\title{
PARAMETRIZATIONS OF ANALYTIC VARIETIES
}

\author{
BY \\ JOSEPH BECKER( $\left.{ }^{1}\right)$
}

ABSTRACT. Let $V$ be an analytic subvariety of an open subset $\Omega$ of $\mathrm{C}^{n}$ of pure dimension $r$; for any $p \in V$, there exists an $n-r \operatorname{dim}$ plane $T$ such that $\pi_{T}: V \rightarrow \mathrm{C}^{r}$, the projection along $T$ to $\mathrm{C}^{r}$, is a branched covering of finite sheeting order $\mu(V, p, T)$ in some neighborhood of $V$ about $p . \pi_{T}$ is called a global parametrization of $V$ if $\pi_{T}$ has all discrete fibers, e.g. $\operatorname{dim}_{p} V \cap$ $(T+p)=0$ for all $p \in V$.

Theorem. $B=\left\{(p, T) \in V \times G(n-r, n) \mid \operatorname{dim}_{p} V \cap(T+p)>0\right\}$ is an analytic set. If $\pi_{2}: V \times G \rightarrow G$ is the natural projection, then $\pi_{2}(B)$ is a negligible set in $G$.

Theorem. $\{(p, T) \in V \times G \mid \mu(V, p, T) \geq k\}$ is an analytic set. For each $p \in V$, there is a least $\mu(V, p)$ and greatest $m(V, p)$ sheeting multiplicity over all $T \in G$.

If $\Omega$ is Stein, $V$ is the locus of finitely many holomorphic functions but its ideal in $\mathcal{O}(\Omega)$ is not necessarily finitely generated.

Theorem. If $\mu(V, p)$ is bounded on $V$, then its ideal is finitely generated.

The purpose of this paper is to extend the theory of local and global parametrizations of analytic varieties and give applications to the global theory of several complex variables.

In the local theory of analytic varieties, great use is made of the local parametrization theorem which says that locally a subvariety $V$ of $\operatorname{dim} r$ can be mapped into $\mathbf{C}^{r}$ by a finite-to-one linear function. Questions about varieties can thus be reduced to related questions about holomorphic functions on $\mathrm{C}^{r}$. A projection $\mathrm{C}^{n} \rightarrow \mathrm{C}^{r}$ (with respect to some basis of $\mathrm{C}^{n}$ ) is called a local parametrization of $V$ at $p$, if there exists a neighborhood $N$ of $p$ such that $\pi \mid V \cap N$ is proper with finite fibers. In $\S 1$, several other algebraic and geometric equivalent definitions are given.

A projection $\pi: \mathbf{C}^{n} \rightarrow \mathrm{C}^{r}$ is called a global parametrization if $\pi \mid V$ has discrete fibers. Any projection $\pi: \mathrm{C}^{n} \rightarrow \mathrm{C}^{n-k}$ can be represented by an element $T \in G(k, n)$, the Grassmann manifold of $k$-dim planes in $\mathbf{C}^{n}$, by projection along $T$ to a complementary subspace. The fibers of $\pi \mid V$ and $\pi^{-1}(p)=V \cap(T+p)$.

Theorem. $B_{j}=\left\{(p, T) \in V \times G(k, n) \mid \operatorname{dim}_{p}(V \cap(T+p)) \geq j\right\}$ is an analytic

Received by the editors November 6, 1972.

AMS (MOS) subject classifications (1970). Primary 32B10, 32C25.

Key words and phrases. Bounded multiplicity, finitely generated ideal.

(1) Supported by National Science Foundation Grant GU 3171. 
subvariety of $V \times G(k, n)$. If $\pi_{2}: V \times G(k, n) \rightarrow G(k, n)$ is the natural projection, then $\pi_{2}\left(B_{j}\right)$ is the countable union of local varieties of codimension at least $j(n-r-k+j)$. It is not always possible to write this as the union of finitely many local varieties, but it is possible if $V$ is an algebraic variety.

A global parametrization is determined by any $T \in G(n-r, n)$ such that $\operatorname{dim}_{p}(V \cap(T+p))=0$ for all $p \in V$, in which case we say $T$ is good for $V$. Considering the case $j=1$ and $k=n-r$, we see that the set of good $T$ forms a second category set in $G(n-r, n)$.

One might attempt to develop some more of the global theory of several complex variables as in Bishop's papers ([2], [3], [4]) if it were possible to find a linear map $\pi: \mathrm{C}^{n} \rightarrow \mathrm{C}^{r}$ expressing $V$ as a countable increasing union of analytic covers. It is possible to do this using a holomorphic map, but this is insufficient for some applications. The best $I$ have been able to do is to show the existence of a linear map $\pi$ such that $\pi \mid V$ has discrete fibers. This result has applications to the extension of analytic varieties [1].

A global parametrization of a variety of pure $\operatorname{dim} r$ is locally an analytic cover of sheeting multiplicity $\mu(V, p, T)$ near $p$.

Theorem. $\{(p, T) \in V \times G(n-r, n) \mid \mu(V, p, T) \geq k\}$ is an analytic subvariety of $V \times G(n-r, n)$. For each $p \in V$, there is a least and greatest multiplicity, $\mu(p, V)$ and $m(p, V)$ respectively, over all good $T \in G(n-r, n)$.

An important result in the global theory of analytic varieties is that any subvariety $V$ of $\mathbf{C}^{n}$ is the common locus of all holomorphic functions vanishing on it. Even more is true $-V$ can be written as the locus of finitely many such functions; indeed, Forster and Ramspott have shown that any subvariety of an $n$-dimensional Stein space is the locus on $n$ functions [5]. However the ideal of all holomorphic functions vanishing on $V$ is not necessarily finitely generated. In $\$ 5$, I give a geometric condition which implies the ideal is finitely generated-if the minimal multiplicity $\mu(V, p)$ is bounded on $V$.

This paper was the author's Ph.D. thesis at Rice University. The author would like to thank his advisor, R. O. Wells, Jr., and Reese Harvey for their constant help and encouragement, and R. C. Gunning and H. Whitney for useful correspondence.

1. Local parameterization of varieties. The results of this section are well known and can be found in [6], [7], and [11]. They are collected together for later reference.

Let $\mathrm{C}^{n}$ denote complex $n$-space. For $a \in \mathbf{C}^{n}$, let ${ }_{n} \mathcal{O}_{a}$ denote the ring of germs of functions holomorphic in a neighborhood of $a$. 
Let $I$ be an ideal of ${ }_{n} \mathcal{O}_{0}, 0 \neq I \neq{ }_{n} \mathcal{O}_{0}, 0 \leq p \leq n$, and $\left(z_{1}, \cdots, z_{n}\right)$ coordinates in $\mathrm{C}^{n}$ with respect to an ordered basis $e_{1}, \cdots, e_{n}$. Then we have a natural homomorphism of rings ${ }_{p} \mathcal{O}_{0} \rightarrow{ }_{n} \mathcal{O}_{0} / l$. It is clear that it is monic if and only if ${ }_{p} \mathcal{O}_{0} \cap I=\{0\}$.

Theorem. The following conditions are equivalent. If any one of them bolds, $\left(z_{1}, \cdots, z_{p}, \cdots, z_{n}\right)$ is said to be a semiregular coordinate system for I.

(1) There exist Weierstrass polynomials

$$
P_{r}\left(z_{1}, \cdots, z_{r-1} ; z_{r}\right) \epsilon_{r-1} \Theta_{0}\left[z_{r}\right] \cap I
$$

for each $r, p+1 \leq r \leq n$, and ${ }_{p} \mathcal{\Theta}_{0} \cap I=\{0\}$.

(2) ${ }_{n} \Theta_{0} / I$ is a finitely generated ${ }_{p} \Theta_{0}$ module and ${ }_{p} \Theta_{0} \rightarrow{ }_{n} \Theta_{0} / I$ is monic.

(3) ${ }_{n} \mathcal{O}_{0} / I$ is integral over ${ }_{p} \mathcal{\Theta}_{0}$ and ${ }_{p} \mathcal{\Theta}_{0} \rightarrow{ }_{n} \mathcal{\Theta}_{0} / I$ is monic.

(4) There exist Weierstrass polynomials.

$$
P_{r}\left(z_{1}, \cdots, z_{p} ; z_{r}\right) \in{ }_{p} \mathcal{O}_{0}\left[z_{r}\right] \cap I
$$

for each $r, p+1 \leq r \leq n$, and ${ }_{p} \mathcal{O}_{0} \cap I=\{0\}$.

(5) ${ }_{p} \Theta_{0} \cap I=\{0\}$ and the ideal of ${ }_{n} \Theta_{0}$ generated by $I$ and $z_{1}, \cdots, z_{p}$ defines the point germ $\{0\}$ at the origin. ( 0 is an isolated point of $\left(O_{p} \times \mathbf{C}^{n-p}\right) \cap \underline{V}(I)$.)

(6) ${ }_{p} \mathcal{O}_{0} \cap I=\{0\}$ and for each $r, p+1 \leq r \leq n$, the ideal of ${ }_{r} \mathcal{O}_{0}$ generated by ${ }_{r} \mathcal{O}_{0} \cap I$ and $z_{1}, \cdots, z_{p}$ defines the point germ $\{0\}$ in $\mathrm{C}^{r}$ at the origin. ( 0 is an isolated point of $\left(O_{p} \times \mathbf{C}^{r-p}\right) \cap \underline{V}\left(I \cap{ }_{r} \Theta_{0}\right)$, where $\underline{V}$ is the locus in $\mathrm{C}^{r}$ of the ideal $I \cap{ }_{r} \mathcal{O}_{0}$ of ${ }_{r} \Theta_{0}$.)

(7) $H=O_{p} \times \mathbf{C}^{n-p} \subset \mathrm{C}^{n}$ is the maximal linear subspace of $\mathrm{C}^{n}$ satisfying $H \cap \underline{V}(I)$ is a point germ, i.e., if $L$ is a linear subspace of $\mathrm{C}^{n}$ with $L \supset H$ and $L \cap \underline{V}(I)$ a point germ, then $H=L$.

The proof $(1) \rightarrow(2)$ is given here as it will be needed in the last section of this paper. Let $q_{r}=$ degree in $z_{r}$ of $P_{r}$. Given $f \in{ }_{n} \Theta$, since $P_{n}$ does not vanish identically in $z_{n}$ direction, the division the orem implies $f=g_{n} P_{n}^{n}+\sum_{i=0}^{q_{n}-1} b_{n, i} z_{n}^{i}$ with $b_{n, i} \in{ }_{n-1} \mathcal{O}$ and $g_{n} \in{ }_{n} \mathcal{O}$. Since $P_{n} \in I$, we can write $f=\Sigma b_{n, i} z_{n}^{i} \bmod I$. Now apply division the orem in ${ }_{n-1} \mathcal{O}$ for each $b_{n, i}$

$$
b_{n, i}=g_{n-1, i} P_{n-1}+\sum_{j=0}^{q}{ }_{n-1}^{-1} b_{n-1, i, j} z_{n-1}^{j} \text { with } b_{n-1, i, j} \epsilon_{n-2} \Theta .
$$

Continue until the pth stage, substituting in the first equation

$$
f=\sum_{a_{r}<q_{r}} f_{a\left(z_{1}, \cdots, z_{p}\right)^{z_{p+1}^{p+1}} \cdots z_{n}^{a_{n}} \bmod I}
$$


with $f_{a} \in{ }_{p} \Theta$. Thus ${ }_{n} \Theta / l$ is generated by the monomials $z_{p+1}^{a}{ }_{p+1} \cdots z_{n}^{{ }^{n}{ }_{n}}$ with $a_{r}<q_{r}$

Remark. Note that it was not necessary for the polynomials $P_{r}$ to be Weierstrass.

Definition. If $\underline{V}$ is an analytic germ, $\left(z_{1}, \cdots, z_{n}\right)$ is said to be semiregular for $\underline{V}$ if it is semiregular for the ideal $I(\underline{V})$.

There is another equivalent condition in terms of this geometric definition depending upon the fact that if the projection $\pi_{r}: \mathrm{C}^{n} \rightarrow \mathrm{C}^{r}$ restricted to $\underline{V}$ has discrete fibers, then $\pi_{r}(\underline{V})$ is an analytic germ.

(8) $O_{p} \times \mathbf{C}^{n-p}$ is semiregular for $\underline{V}$ if and only if $O_{p} \times \mathbf{C}^{r-p}$ is semiregular for $\pi_{r}(\underline{V})$ for all $r, p+1 \leq r \leq n$.

This follows immediately from condition (6) since $\underline{V}\left(I \cap{ }_{r} \Theta_{0}\right)=\pi_{r}(\underline{V})$. Clearly $I \cap{ }_{r} \Theta_{0}=I\left(\pi_{r}(\underline{V})\right)$, the ideal in ${ }_{r} \Theta_{0}$ of functions vanishing on $\pi_{r}(V)$. Hence $\underline{V}\left(I \cap{ }_{r} \mathcal{\Theta}_{0}\right)=\underline{V}\left(I\left(\pi_{r}(\underline{V})\right)\right)=\pi_{r}(V)$, since $\pi_{r}(\underline{V})$ is analytic.

If $\left(z_{1}, \cdots, z_{p}, \cdots, z_{n}\right)$ is semiregular for $\underline{V}$, the projection $\pi: V \rightarrow \mathrm{C}^{p}$ is called a local parameterization of the variety. It is easy to show from definition (1) that such coordinate systems exist and $\operatorname{dim} \underline{V}=p$. So far we have only defined semiregular coordinates for the germ of a variety at the origin-the extension of this definition is that $\left(z_{1}, \cdots, z_{n}\right)$ are semiregular for an analytic germ $\underline{V}_{a}$ if they are semiregular for $\underline{V}_{a}+(-a)=\left\{z-a \mid z \in \underline{V}_{a}\right\}$. The question then arises that, if $V$ is a subvariety of $\operatorname{dim} p$ of an open subset of $\mathbf{C}^{n}$, do there exist coordinates which are semiregular at every point of $V$. The answer is yes as we will see below.

Given a basis $e_{1}, \cdots, e_{n}$ of $\mathrm{C}^{n}$, the definition of ${ }_{r} \mathcal{O}_{0}$ depends only upon the linear span of $e_{r+1}, \cdots, e_{n}$. Thus it is clear from conditions (2), (3), and (7) that the statement that $\left(z_{1}, \cdots, z_{p}, \cdots, z_{n}\right)$ is semiregular depends only upon the span of $e_{p+1}, \cdots, e_{n}$. Let $G(k, n)$ be the Grassmann manifold of $k$ complex dimensional linear subspaces of $\mathrm{C}^{n}$. Then $G(k, n)$ is a compact complex manifold of dimension $k(n-k)$. Let $\underline{V}$ be an analytic germ of $\operatorname{dim} p$; an element $T \in G(n-p, n)$ is said to be good for $\underline{V}$ if $T \cap \underline{V}=\{0\}$ and $T$ is said to be bad if it is not good.

\section{Global coordinates for $\mathbf{v}$ arieties.}

2.1. Let $V$ be a subvariety of dimension $r$ of an open subset $\Omega$ of $\mathbf{C}^{n}, B_{j}=$ $\left\{(p, T) \in V \times G(k, n) \mid \operatorname{dim}_{p}(V \cap(T+p)) \geq j\right\}$, and $\pi_{2}: V \times G \rightarrow G$ and $\pi_{1}: V \times G \rightarrow$ $V$ the natural projections. In [8, Sätze 9-11], Grauert proved that for $j=1$ and $k=n-r, \pi_{2}\left(B_{1}\right)=\{T \in G \mid T$ bad at some $p \in V\}$ is a first category set in $G(n-r, n)$. In this section we improve this result by showing that $\pi_{2}\left(B_{j}\right)$ is the countable union of local $\mathrm{v}$ arieties of codimension at least $j(n-k-r+j)$ in $G(k, n)$. This is the best possible estimate on the codimension since if $V=\mathbf{C}^{r}$, 
any element $T \in \pi_{2}\left(B_{j}-B_{j+1} \cup \cdots \cup B_{k}\right)$ can be written uniquely as the direct sum of a $j$-dim plane $T^{\prime \prime}$.in $C^{r}$ and $k-j \operatorname{dim}$ plane $T^{\prime}$ in a fixed $n-j \operatorname{dim}$ complementary space to $T^{\prime \prime}$. Hence $\operatorname{dim} \pi_{2}\left(B_{j}\right) \geq j(r-j)+(k-j)(n-k) \operatorname{socodim} \pi_{2}\left(B_{j}\right)$ in $G(k, n) \leq j(n-r-k+j)$.

Proposition 2.1. Let $V$ be a subvariety of an open subset of $\mathrm{C}^{n}$ and $B_{j}=$ $\left\{(p, T) \in G(k, n) \mid \operatorname{dim}_{p}(V \cap(T+p)) \geq j\right\}$. Then $B_{j}$ is an analytic set in $V \times G(k, n)$.

Proof. For each subset of $n-k$ elements, $H \subset\{1,2, \cdots, n\}$, define a map $\mathrm{C}^{n-k} \rightarrow \mathrm{C}^{n}$ by placing 0 in each coordinate not in $H$. The images of these maps will be called the canonical copies of $\mathrm{C}^{n-k}$ in $\mathrm{C}^{n}$. For each canonical $\mathrm{C}^{n-k}$ in $\mathrm{C}^{n}$, consider the set $U=\left\{T \in G(k, n) \mid T \cap \mathrm{C}^{n-k}=\{0\}\right\}$. These form a covering of $G(k, n)$ and we show that $B \cap(V \times U)$ is analytic in $V \times U$. (For convenience, assume $\mathrm{C}^{n-k}$ is the last $n-k$ coordinates.)

Define a holomorphic map $f: \mathrm{C}^{n} \times U \rightarrow \mathrm{C}^{n-k}$ so that, for each fixed $T$, the map is projection along $T$ to $\mathrm{C}^{n-k}$. Let $T \in U$ be spanned by the $k$ row vectors $\left(a_{j 1}, a_{j 2}, \cdots, a_{j n}\right)$ for $1 \leq j \leq k$, and $A$ be the $n \times n$ matrix

$$
\left[\begin{array}{lll}
a_{11} & a_{12} & a_{1 n} \\
\vdots & & \\
a_{k 1} & a_{k 2} & a_{k n} \\
& & \\
& 0_{n-k \times n} & I_{n-k}
\end{array}\right]
$$

If $z_{1}, \cdots, z_{n}$ are the coordinates of a point with respect to the basis $e_{1}, \cdots, e_{n}$ and $w_{1}, \cdots, w_{n}$ are the coordinates $w$ ith respect to the basis given by the rows of $A$, then $\left(w_{1}, \cdots, w_{n}\right)=\left(z_{1}, \cdots, z_{n}\right) A^{-1}$. The map $f$ is given by $f\left(z_{1}, \cdots, z_{n}, a_{i j}\right)=\left(w_{n-k+1}, \cdots, w_{n}\right)$. Now define $\pi: V \times U \rightarrow \mathrm{C}^{n-k} \times G$ by $\pi(p, T)=(f(p, T), T)$; the fiber $\pi^{-1} \pi(p, t)=V \cap(T+p) \times T$. Thus

$$
\left\{(p, T) \in V \times U \mid \operatorname{dim}_{p}(V \cap(T+p)) \geq j\right\}=\left\{(p, T) \in V \times U \mid \operatorname{dim}_{\left.(p \cdot T)^{\pi^{-1}} \pi(p, T) \geq j\right\}}\right.
$$

is analytic. (By a theorem of Remmert [12, Satz 17] which says that if $\pi: X \rightarrow Y$ is any holomorphic map between complex spaces, then $\left\{x \in X \mid \operatorname{dim}_{x} \pi^{-1} \pi(x) \geq k\right\}$ is an analytic set.)

Lemma 2.2. If $\operatorname{dim} V=r$ and $B_{k} \subset V \times G(k, n)$, then $\operatorname{rank} \pi_{2} \mid B_{k} \leq k(r-k)$.

Proof. Let $m=\operatorname{rank} \pi_{2} \mid B_{k}$ and suppose $m>k(r-k)$. There exists a regular 
point of $B_{k}$ where the Jacobian rank of $\pi_{2}$ is $m$. By the implicit function theorem, we may assume $\pi_{2}$ is of the form $\left(z_{1}, \cdots, z_{\rho}\right) \rightarrow\left(z_{1}, \cdots, z_{m}, 0, \cdots, 0\right)$, after a local biholomorphic change of coordinates in $B_{k}$ and $G(k, n)$. Hence there is a holomorphic section $g$ of $\pi_{2}$ over some $m$-dim submanifold $M$ of an open subset of $G(k, n)$. Define a map $G: M \times \mathbf{C}^{k} \rightarrow \mathbf{C}^{n} \times G(k, n)$ by

$$
G\left(z_{1}, \cdots, z_{m}, \alpha_{1}, \cdots, \alpha_{k}\right)=\left(\phi+\alpha_{1} \Psi_{1}+\cdots+\alpha_{k} \Psi_{k}, \pi_{2} g\right)
$$

where $\phi\left(z_{1}, \cdots, z_{m}\right)=\pi_{1}(g)$ and $\Psi\left(z_{1}, \cdots, z_{m}\right)=\left(\Psi_{1}, \cdots, \Psi_{k}\right) ; M \rightarrow C^{k n}$ is the lifting of a $k$ plane to a basis of the plane. Then $U=G^{-1}(\Omega \times G(k, n))$ is open in $M \times C^{k}$ and $G(U) \subset B$ by construction. We show that the map $F=\pi_{1} G=$ $\phi+a_{1} \Psi_{1}+\cdots+a_{k} \Psi_{k}: U \rightarrow V$ has Jacobian rank $>r$, contradicting the fact that $\operatorname{dim} V=r$.

Now $\psi: M \rightarrow \mathrm{C}^{k n}$ has rank $m$ and $\psi_{i}$ is the $i$ th column of the $n \times k$ matrix

$$
\left[\psi_{i j}\right]=\left[\begin{array}{ccccc}
\Psi_{11} & \Psi_{12} & & & \Psi_{1 k} \\
\Psi_{21} & \Psi_{22} & & & \Psi_{2 k} \\
\Psi_{n-k, 1} & \Psi_{n-k, 2} & & & \Psi_{n-k, k} \\
1 & 0 & 0 & & 0 \\
0 & 1 & 0 & \ldots & 0 \\
0 & 0 & 1 & \cdots & \\
\vdots & & & & 0 \\
0 & & 0 & & 1
\end{array}\right] .
$$

The $n \times(m+k)$ Jacobian matrix of $F$ is

$$
\left[\frac{\partial \phi_{i}}{\partial z_{b}}+\sum_{j=1}^{k} \alpha_{j} \frac{\partial \Psi_{i j}}{\partial z_{b}}: \Psi\right]
$$

It suffices to show that in the expansion of some $r+1$ subdeterminant as a polynomial in $\alpha_{1}, \cdots, \alpha_{k}$, the coefficient of the highest degree monomial is nonzero. To do this, it suffices to assume our matrix is

$$
C=\left[\sum_{j=1}^{k} a_{j} \frac{\partial \Psi}{\partial z_{b}}: \Psi\right]
$$


This matrix is of the form

$$
\left[\begin{array}{ll}
A_{n-k \times m}^{\prime} & * \\
0_{k \times m} & I_{k}
\end{array}\right],
$$

so that rank $A^{\prime}>r-k$ if and only if the column rank of $C>r$. To see the row rank of $A^{\prime}>r-k$, recall that the $k(n-k) \times m$ matrix $A=\left[\partial \Psi_{i j} / \partial z_{b}\right]$ for $1 \leq i \leq n-k, 1 \leq j \leq k, 1 \leq b \leq m$, whose $k(i-1)+j$ th row $v_{i j}$ is $\left(\partial \Psi_{i j} / \partial z_{1}, \cdots\right.$, $\left.\partial \Psi_{i j} / \partial z_{m}\right)$, has rank $m$. Note that the $i$ th row of $A^{\prime}$ is $\sum_{j=1}^{k} \alpha_{j} v_{i j}$. Now elementary column operations do not change the row rank of $A^{\prime}$ and a column operation on $A$ induces a corresponding column operation on $A^{\prime}$ (but a row operation on $A$ does not induce a row operation on $A^{\prime}$ ), so we can assume $A$ is in column echelon form.

$$
\left[\begin{array}{cccc}
I_{i_{1}} & 0 & & 0 \\
* & 0 & & 0 \\
0 & I_{i_{2}} & 0 & 0 \\
* & * & 0 & 0 \\
0 & 0 & I_{i_{3}} & \\
& & & 0 \\
0 & & & I_{i_{q}}
\end{array}\right], \quad i_{1}+i_{2}+\cdots+i_{q}=m .
$$

Since $m>k(r-k)$, there are at least $r-k+1$ different values of $i, 1 \leq i \leq n-k$, such that there exist $j$ and $b, 1 \leq j \leq k, k(i-1)+1 \leq b \leq k i$, with $\partial \Psi_{i j} / \partial z_{b} \neq 0$. For any such $i$, the set of $L_{i}$ of $\left(a_{1}, \cdots, a_{k}\right)$ satisfying $\bar{\Sigma}_{j=1}^{k} a_{j} \partial \Psi_{i j} / \partial z_{b}=0$ for all $k(i-1)+1 \leq b \leq k i$ is a proper linear subspace of $\mathbf{C}^{k}$. Then for $\left(a_{1}, \cdots\right.$, $\left.a_{k}\right) \notin \bigcup_{L_{i}}$, it is easily seen that row rank $\left(A^{\prime}\right) \geq r-k+1$.

Theorem 2.3. Let $\operatorname{dim} V=r$ and $A_{j}=\left\{(p, T) \in V \times G(k, n) \mid \operatorname{dim}_{p}(V \cap(T+p))=\right.$ j\}. Then $\operatorname{rank} \pi_{2} \mid A_{j} \leq j(r-j)+(k-j)(n-k)$.

Proof. This result has already been shown for $k=j$, so we need only to reduce the problem to this case. We will take an open covering of $V \times G(k, n)$ and show the result for the restriction of $\pi_{2}$ for each element of the covering. For each canonical $\mathrm{C}^{n-k}$ in $\mathrm{C}^{n}$, recall $U=\{T \in G(k, n) \mid T$ is transversal to $\left.\mathrm{C}^{n-k}\right\}$. Let $\left(p_{0}, T_{0}\right) \in A_{j}$ and write $T_{0}$ as the direct sum of subspaces $T_{0}^{\prime}$ and 
$T_{0}^{\prime \prime}$ with $\operatorname{dim} T_{0}^{\prime}=k-j$ and $\operatorname{dim} T_{0}^{\prime \prime}=j$ such that the projection of $T_{0}+p_{0}$ to $T_{0}^{\prime \prime}+$ $p_{0}$ along $T_{0}^{\prime}$ when restricted to $V$ has discrete fibers in a neighborhood of $p_{0}$. Now take a basis $e_{1}, \cdots, e_{n}$ of $\mathrm{C}^{n}$ such that $e_{1}, \cdots, e_{k-j} \operatorname{span} T_{0}^{\prime}, e_{k-j+1}, \cdots, e_{k}$ span $T_{0}^{\prime \prime}$, and $e_{k+1}, \cdots, e_{n}$ span $\mathrm{C}^{n-k}$; let $\mathrm{C}^{n-k+j}$ denote the span of $e_{k-j+1}, \cdots, e_{n}$.

Each $T \in U$ is represented as a $k \times n$ matrix $\left[P_{k \times k}: Q_{k \times k}\right]$ with $P$ nonsingular since $T$ is transversal to $\mathrm{C}^{n-k}$. Now applying the canonical coordinate systems on the Grassmann, we see that $T$ can be represented by

$$
\left[\begin{array}{cccc}
I_{k-j} & 0 & : & \\
0 & I_{j} & : & P^{-1} Q
\end{array}\right]
$$

and that $\operatorname{dim}\left(T \cap \mathbf{C}^{n-k+j}\right)=j$. Let $T^{\prime}$ be the space spanned by the first $k-j$ rows of this matrix and $T^{\prime \prime}$ the space spanned by the last $j$ rows. Note that $T^{\prime \prime} \oplus \mathbf{C}^{n-k}=\mathbf{C}^{n-k+j}$ and $T^{\prime \prime}=T \cap \mathbf{C}^{n-k+j}$. Let $G^{\prime}(k-j, n-j)$ be the subset of $G(k-j, n)$ of elements of the form $\left[I_{k-j} \vdots 0_{k-j \times j} \vdots *\right]$. Then there are holomorphic maps

$$
\begin{array}{ll}
U \rightarrow G(j, n-k+j), & U \rightarrow G^{\prime}(k-j, n-j), \\
T \rightarrow T^{\prime \prime}, & T \rightarrow T^{\prime} \text { of rank } \leq(k-j)(n-k)
\end{array}
$$

and

$$
\begin{array}{r}
\sigma: G(j, n-k+j) \times G^{\prime}(k-j, n-j) \rightarrow G(k, n), \\
\left(T^{\prime \prime}, T^{\prime}\right) \rightarrow T^{\prime \prime}+T^{\prime} .
\end{array}
$$

Define a holomorphic map $\pi: \mathrm{C}^{n} \times U \rightarrow \mathrm{C}^{n-k+j}$ so that for each fixed $T$, the map is projection along $T^{\prime}$ to $\mathrm{C}^{n-k+j}$. Let $M$ be the matrix

$$
\left[\begin{array}{ll}
I_{k} & P^{-1} Q \\
0 & I_{n-k}
\end{array}\right] \text {. }
$$

If $z_{1}, \cdots, z_{n}$ are the coordinates of a point in terms of the basis $e_{1}, \cdots, e_{n}$ and $w_{1}, \cdots, w_{n}$ are the coordinates in terms of the basis given by the rows of $M$, then $\left(w_{1}, \cdots, w_{n}\right)=\left(z_{1}, \cdots, z_{n}\right) M^{-1}$. Let $\pi\left(z_{1}, \cdots, z_{n}, T\right)=\left(w_{k-j+1}, \cdots, w_{n}\right)$. Now define a map $A_{j} \cap(V \times U) \rightarrow \mathrm{C}^{n-k+j} \times G(k, n)$ by $(p, T) \rightarrow(\pi(p, T), T)$. The fibers of this map are $V \cap\left(T^{\prime}+p\right) \times T$. By construction, the fiber at $\left(p_{0}, T_{0}\right)$ is of dimension zero. By semicontinuity, there exists a neighborhood which we 
again denote $V \times U$ of $\left(p_{0}, T_{0}\right)$ in $A_{j} \cap(V \times U)$ such that the restriction to $V \times U$ has fibers of dimension zero. For $(p, T),(p, S) \in V \times U$ with $T^{\prime}=S^{\prime}$, $\operatorname{dim}_{p}\left(V \cap\left(S^{\prime}+p\right)\right)=0$ so $\pi_{T}: V \cap(S+p) \rightarrow S^{\prime \prime}+p$ has discrete fibers. Thus $\operatorname{dim}_{p} \pi_{T}(V) \cap\left(S^{\prime \prime}+p\right)=\operatorname{dim}_{p} \pi_{T}(V \cap(S+p))=j$. Now let $A_{j}^{\prime}=\{(p, T, S) \epsilon$ $V \times U \times U \mid(p, T),(p, S) \in A_{j}$ and $\left.T^{\prime}=S^{\prime}\right\}$. Then $A_{j}^{\prime}$ is a local variety. Consider the following diagram

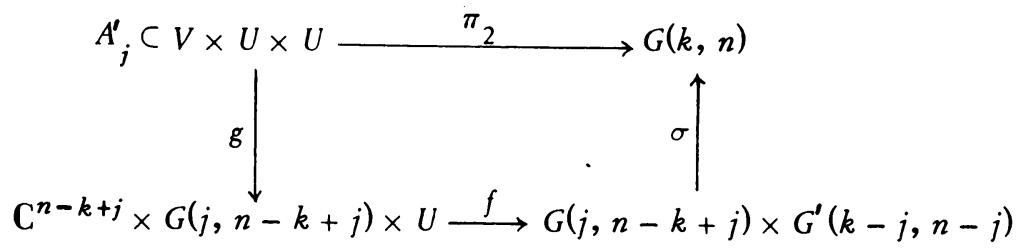

$\pi_{2}(p, T, S)=T, g(p, T, S)=\left(\pi(p, T), S^{\prime \prime}, T\right), f\left(q, S^{\prime \prime}, T\right)=\left(S^{\prime \prime}, T^{\prime}\right)$. Note that $\sigma f g(p, T, T)=T^{\prime \prime}+T^{\prime}=T=\pi_{2}(p, T, T)$ so $\sigma$ is onto the image of $\pi_{2}$. It suffices to give an upper bound for the rank of $f$. The fibers of $g, g^{-1} g(p, T, S)=$ $V \cap\left(T^{\prime}+p\right) \underset{\sim}{\times} T \times S$ are of dimension zero so in some neighborhood of $\underset{\sim}{\sim} p_{0}, T_{0}$, $\left.T_{0}\right), g\left(A_{j}^{\prime}\right)=\widetilde{A}_{j}$ is an analytic germ. As previously noted, $\left(q, S^{\prime \prime}, T\right) \in \tilde{A}_{j} \Rightarrow$ $\operatorname{dim}_{q} \pi_{T}(V) \cap\left(S^{\prime \prime}+q\right)=j$ so since $\operatorname{dim} \pi_{T}(V)=r$, for fixed $T$, the rank of $\tilde{A}_{j} \stackrel{\stackrel{q}{\prime \prime}^{\prime \prime}}{\rightarrow} G(j, n-k+j)$ is at most $j(r-j)$, and the rank of $\tilde{A}_{j} \stackrel{T}{\prime}^{\prime} G^{\prime}(k-j, n-j)$ is at most $(k-j)(n-k)$. Thus $\operatorname{rank}\left(f \mid \tilde{A}_{j}\right) \leq j(r-j)+(k-j)(n-k)$.

Corollary 2.4. If $\operatorname{dim} V=r$, then $\pi_{2}\left(B_{j}\right)$ is the countable union of local varieties of codimension at least $j(n-r-k+j)$ in $G(k, n)$.

Proof. $B_{j}$ is the disjoint union of $A_{j}, \cdots, A_{k}$ and $\operatorname{codim} \pi_{2}\left(A_{l}\right) \geq k(n-k)-$ $[l(r-l)+(k-l)(n-k)]=l(n-r-k+l)$ so $\operatorname{codim} \pi_{2}\left(B_{j}\right)=\min _{j \leq l \leq k} l(n-r-k+l)=$ $j(n-r-k+j)$.

2.2. Above we show that $B_{j}$ is an analytic set in $V \times G(k, n)$; we will now explicitly give the equations whose locus in $V \times G(k, n)$ is $B_{j}$.

First consider the special case $j=k=1$ and $r=n-1$. Let $p \in V$ and in some neighborhood of $p, V$ is the locus of $f(z)=\Sigma_{a} f_{a}(p)(z-p)^{a}$, a multi-index. Now $a=\left[a_{1}, \cdots, a_{n}\right] \in \mathbf{C P}^{n-1}$ is bad for $V$ at $p$ if and only if for all small $\lambda \in \mathbf{C}$

$$
0=f(p+\lambda a)=\sum_{a} f_{\alpha}(p)(\lambda a)^{a}=\sum_{k=0}^{\infty} \lambda^{k} \sum_{|a|=k} f_{a}(p) a^{a}
$$

if and only if $0=\Sigma_{|a|=k} f_{\alpha}(p) a^{\alpha}$ for all $k$. These analytic equations are homogenous in $a$ for each $p$, so define a subvariety of $V \times \mathbf{C P}^{n-1}$.

More generally, $B_{j}=\left\{(p, T) \in V \times G(k, n) \mid \operatorname{dim}_{p}(V \cap(T+p)) \geq j\right\}$; let $T$ be 
represented by the $k \times n$ matrix $\left[a_{i j}\right]$ with row vectors $a_{1}, \cdots, a_{k}$. Then

$$
B_{j}=\bigcup_{m, H, Q} \bigcap_{l, l_{i}, f} \text { locus of } \sum_{\substack{a_{1}+\cdots+a_{j}=a \\
|\alpha|=l,\left|\alpha_{i}\right|=l}}\left(\begin{array}{c}
a \\
a_{1} \cdots \alpha_{j}
\end{array}\right) f_{\alpha}(p) a_{q_{1}}^{a_{1}} \cdots a_{q_{j}}^{a_{j}}
$$

where $l \geq 0, l_{i} \geq 0, l_{1}+\cdots+l_{j}=l, f \in I\left(\underline{V}_{p}\right), 0 \leq m \leq k-j, H=\left\{b_{1}, \cdots, b_{m}\right\}$ is a subset of $m$ elements of $\{1, \cdots, n\}, Q=\left\{q_{1}, \cdots, q_{j}\right\}$ is a subset of $j$ elements of $\{1, \cdots, n\}, H \cap Q=\varnothing, \alpha$ is a multi-index of length $n$ whose $\left(b_{1}, \cdots, b_{m}\right)$ th coordinates are zero, $\Sigma_{i=1}^{n} a_{b i} \partial f / \partial z_{i} \equiv 0$ for each $b \in H$, and the binomial coefficient $\left(\begin{array}{c}a \\ a_{1} \ldots a_{j}\end{array}\right)=\alpha ! / \alpha_{1} ! \cdots \alpha_{j} !$.

Proof. Suppose $(p, T) \in B_{j}$ and $V \cap(T+p)$ contains a $j$-dim plane near $p$ spanned by $a_{q_{1}}, \cdots, a_{q_{j}}$. Then for all $f \in I_{p}(V)$ and small $\lambda_{1}, \cdots, \lambda_{j} \in \mathrm{C}$

$$
\begin{aligned}
0 & \equiv f\left(p+\lambda_{1} a_{q_{1}}+\cdots+\lambda_{j} a_{q_{j}}\right)=\sum_{a} f_{a}(p)\left(\lambda_{1} a_{q_{1}}+\cdots+\lambda_{j} a_{q_{j}}\right)^{a} \\
& =\sum_{\substack{l_{i}, l \geq 0 \\
l_{1}+\cdots+l_{j}=l}} \lambda_{1}^{l_{1}} \cdots \lambda_{j}^{l_{j}} \sum_{\substack{\left|a_{i}\right|=l_{i} \\
a_{1}+\cdots+a_{j}=a}}\left(\begin{array}{c}
a \\
a_{1} \cdots a_{j}
\end{array}\right) f_{\alpha}(p) a_{q_{1}}^{a_{1}} \cdots a_{q_{j}}^{a_{j}}
\end{aligned}
$$

On the other hand, if $V \cap(T+p)$ does not contain a $j$-dim plane near $p$, there exists a $m-\operatorname{dim}$ plane $T^{\prime}$ in $T, m=k-\operatorname{dim}_{p}(V \cap(T+p))$ spanned by $a_{b_{1}}, \cdots, a_{b_{m}}$ such that $V \cap\left(T^{\prime}+p\right)$ is discrete. Let the projection $\pi: \mathrm{C}^{n} \rightarrow \mathrm{C}^{n-m}$ be defined by $T^{\prime}$; then $\pi \mid V$ has discrete fibers and the analytic germ $\pi\left(\underline{V}_{p}\right)$ contains $j$-dim plane near $p$. Hence the above equations hold in $\mathrm{C}^{n-m}$ for $f \in I\left(\pi\left(\underline{V}_{p}\right)\right)=$ $I\left(\underline{V}_{p}\right) \cap{ }_{n-m} \mathcal{O}_{p}$. The condition that $f$ be independent of the variables $a_{b_{1}}, \cdots$, $a_{b_{m}}$ is precisely the equations $\sum_{i=1}^{n} a_{b i} \partial f / \partial z_{i} \equiv 0$.

\section{Multiplicities of varieties.}

3.1. It is a basic result in the local theory of analytic varieties [7] that if $V$ is an analytic subvariety of an open subset of $\mathbf{C}^{n}$ of $\operatorname{dim} r$ and $T$ is good for $V$ at $p$, then there is a neighborhood $N$ of $p$ in $\mathrm{C}^{n}$ and $N^{\prime}$ open in $\mathrm{C}^{r}$ such that $\pi_{T}$ : $N \cap V \rightarrow N^{\prime}$ is an analytic cover of order $k$, for some integer $k>0$. That is, $\pi_{T}$ is a proper continuous map with finite fibers and there is a hypersurface $W \subset N^{\prime}$ such that $N \cap V-\pi_{T}^{-1}(W)$ is dense in $V$ and $\pi_{T}: N \cap V-\pi_{T}^{-1}(W) \rightarrow N^{\prime}-W$ is a $k$-sheeted covering map.

Definition. Let $p \in V$ and $T \in G(n-r, n)$; if $T$ is good for $V$ at $p, \pi_{T}: V \rightarrow \mathbf{C}^{r}$ is locally an analytic cover near $p$ of sheeting order $\mu(V, p, T)$, the multiplicity of $V$ at $p$ relative to $T$. If $T$ is bad for $V$ at $p$, we set $\mu(V, p, T)=\infty$.

Theorem 3.1. $M_{k}=\{(p, T) \in V \times G(n-r, n) \mid \mu(V, p, T) \geq k\}$ is an analytic subset of $V \times G(n-r, n)$. 
Corollary 3.2. The maximum multiplicity is bounded on compact sets. More precisely, if $K$ is a compact subset of $V$, then $\sup _{p} \in K \sup _{T \operatorname{good} \text { at } p} \mu(V, p, T)<\infty$. $\infty$. In particular, for each $p \in V$, there is a finite maximum multiplicity $m(V, p)=$ $\sup _{T \text { good at } p} \mu(V, p, T)$.

Proof. $B=\bigcap_{k=1}^{\infty} M_{k}$ by definition; $B \cap(K \times G)$ and each $M_{k} \cap(K \times G)$ lie in a compact subset of the complex space $V \times G$ and so have only finitely many irreducible components. Thus the result follows from the lemma below.

Lemma 3.3. Let $A=\bigcap_{i=1}^{\infty} A_{i}$, each $A_{i} \supset A_{i+1}$, where $A$ and each $A_{i}$ are analytic sets with finitely many irreducible components. Then there exists $k$ with $A=A_{k}$.

Proof. Induct on $m=\max \operatorname{dim}$ of irreducible components of $A_{k}$ not in $A$; the conclusion of the lemma holds if $m=-1$. Choose a point $x_{a}$ from each irreducible component of $A_{k}$ not in $A$; then $\left\{x_{a}\right\}$ is a finite set so there exists $k^{\prime}>k$ with $\left\{x_{a}\right\} \cap A_{k}{ }^{\prime}=\varnothing$. Then $m-1 \geq \max \operatorname{dim}$ of irreducible components of $A_{k}^{\prime}$ not in $A$.

In order to prove the main theorem of this section we must first develop some more of the local theory. The first result uses two standard facts concerning the removing of singularities of holomorphic functions.

Rado's theorem. If $U$ is an open set in $\mathbf{C}^{n}$ and $b: U \rightarrow \mathbf{C}$ is a continuous function such that $b$ is bolomorphic on $\{z \in U \mid f(z) \neq 0\}$, then $f$ is bolomorphic on $U$.

Riemann extension theorem. If $U$ is an open set in $\mathbf{C}^{n}, A$ an analytic set in $U$, and $b: U-A \rightarrow \mathrm{C}$ a bounded bolomorphic function, then $b$ extends to a bolomorphic function on $U$.

Proposition 3.4 (Minimal analytic polynomial). Let $V$ be an analytic subvariety of pure $\operatorname{dim} r$ in an open subset $\Omega$ of $\mathrm{C}^{n}, \pi: \mathrm{C}^{n} \rightarrow \mathrm{C}^{r}$ a bolomorphic map, $\Omega^{\prime}=\pi(\Omega)$ and $\pi \mid V$ proper. Then for every $f \in \mathcal{O}(\Omega)$, there is a unique monic pseudopolynomial $P_{f}\left(z^{\prime}, t\right) \in \mathcal{C}\left(\Omega^{\prime}\right)[t]$ with $P_{f}(\pi(z), f(z)) \equiv 0$ on $V$ such that $P_{f}$ has minimal degree with respect to those properties. Furthermore $\left\{t \in \mathrm{C}_{\mathrm{P}} P_{f}\left(z^{\prime}, t\right)=\right.$ $0\}=f\left(\pi^{-1}\left(z^{\prime}\right)\right)$.

Proof. There is a hypersurface $A^{\prime}=\pi\left(\operatorname{Sing}(V) \cup\left\{z \in \operatorname{Reg}(V) \mid \operatorname{rank}_{z} \pi<r\right\}\right)$ in $\Omega^{\prime}$ such that $A=\pi^{-1}\left(A^{\prime}\right)$ is nowhere dense in $V$ and $\pi: V-A \rightarrow \Omega^{\prime}-A^{\prime}$ is a $k$-sheeted covering map. For $z^{\prime} \in \Omega^{\prime}-A^{\prime}$, let $\pi^{-1}\left(z^{\prime}\right)=\left\{w_{1}\left(z^{\prime}\right), \cdots, w_{k}\left(z^{\prime}\right)\right\}$ and $\lambda=$ maximum over all $z^{\prime} \in \Omega^{\prime}-A^{\prime}$ of the number of distinct values among $f w_{1}\left(z^{\prime}\right), \cdots, f w_{k}\left(z^{\prime}\right)$. Let 


$$
b(z)=\left\{\begin{array}{c}
\prod_{1 \leq i \leq j \leq \lambda}\left(f\left(w_{i}\left(z^{\prime}\right)\right)-f\left(w_{j}\left(z^{\prime}\right)\right)\right) \text { when the values are distinct, } \\
0 \text { when there are less than } \lambda \text { distinct values. }
\end{array}\right.
$$

Since $\pi$ is locally biholomorphic over $\Omega^{\prime}-A^{\prime}, b$ is continuous and is holomorphic where it is nonzero. Thus by Rado's theorem, $b$ is holomorphic on $\Omega^{\prime}-A^{\prime}$. Then by the Riemann extension theorem, $b$ is holomorphic on $\Omega^{\prime}$.

Let $B^{\prime}=A^{\prime} \cup\left\{z^{\prime} \in \Omega^{\prime} \mid b\left(z^{\prime}\right)=0\right\}$; for $z^{\prime} \in \Omega^{\prime}-B^{\prime}$, $f$ has $\lambda$ distinct values on $\pi^{-1}\left(z^{\prime}\right)$ so define

$$
P_{f}\left(z^{\prime}, t\right)=\prod_{i=1}^{\lambda}\left(t-f\left(w_{i}\left(z^{\prime}\right)\right)\right)=t^{\lambda}+a_{1}\left(z^{\prime}\right) t^{\lambda-1}+\cdots+a_{\lambda}\left(z^{\prime}\right)
$$

where $a_{i}=\sigma_{i}\left(f w_{1}\left(z^{\prime}\right), \cdots, f w_{\lambda}\left(z^{\prime}\right)\right) \in \Theta\left(\Omega^{\prime}-B^{\prime}\right)$ and the $\sigma_{i}$ are the elementary symmetric functions. Also $a_{i}$ is bounded since $\pi$ is proper, so that $a_{i}$ extend to holomorphic functions on $\Omega^{\prime}$. It is clear that for $z^{\prime} \in \Omega^{\prime}-B^{\prime}$, the roots of $P_{f}\left(z^{\prime}, t\right)$ are precisely the values of $f$ on $\pi^{-1}\left(z^{\prime}\right)$. That this statement also holds for $z^{\prime} \in B^{\prime}$ follows directly from the fact that $\pi$ is proper and $B^{\prime}$ is nowhere dense in $\Omega^{\prime}$.

To show $P_{f}$ is unique, suppose $\left.\tilde{P}\left(z^{\prime}, t\right) \in \mathcal{O}\left(\Omega^{\prime}\right) \underset{\sim}{[}\right]$ is a monic pseudopolynomial of degree $\nu$ and $P(\pi(z), f(z)) \equiv 0$ on $V$. Factor $\widetilde{P}$ into irreducible pseudopolynomials and let $D^{\prime}=$ union of the discriminant loci of the various factors of $\widetilde{P}$. For $z^{\prime} \in \Omega^{\prime}-\left(B^{\prime} \cup D^{\prime}\right)$, we can write by the fundamental theorem of algebra: $\widetilde{P}\left(z^{\prime}, t\right)=\Pi_{j=1}^{\nu}\left(t-r_{j}\left(z^{\prime}\right)\right)$ where the $\lambda$ distinct values $f w_{1}\left(z^{\prime}\right), \cdots, f w_{\cdot \lambda}\left(z^{\prime}\right)$ must occur among the $r_{j}\left(z^{\prime}\right)$. Thus let

$$
\widetilde{P}\left(z^{\prime}, t\right)=Q\left(z^{\prime}, t\right) P_{f}\left(z^{\prime}, t\right)=\prod_{j=\lambda+1}^{\nu}\left(t-r_{j}\left(z^{\prime}\right)\right) \prod_{i=1}^{\lambda}\left(t-f w_{i}\left(z^{\prime}\right)\right) .
$$

Then the coefficients of $Q$ are in $\Theta\left(\Omega^{\prime}-\left(B^{\prime} \cup D^{\prime}\right)\right)$ and as usual extend to $\Omega^{\prime}$. Hence $P_{f}$ divides $\widetilde{P}$ in the ring $O\left(\Omega^{\prime}\right)[t]$ so if $\operatorname{deg} P=\operatorname{deg} P_{f}$, these polynomials are the same.

Remark. If $V$ is a hypersurface, then the minimal analytic polynomial $P_{n}\left(z^{\prime}, z_{n}\right)$ generates the ideal $I(V)$ of all holomorphic functions vanishing on $V$. (See Gunning-Rossi [7, Theorem III-C-11b].) The proof of this statement is actually contained in the previous proposition since any $f \in I(V)$ can be written as a product $g P$ where $P$ is a pseudopolynomial vanishing identically on $V$.

Furthermore if $V$ is not a hypersurface and $V^{\prime}$ is the locus in $\mathrm{C}^{r+1}$ of some $P_{j}\left(z^{\prime}, z_{j}\right), j=r+1, \cdots, n$, then $P_{j}$ generates the ideal of all functions in $r_{r+1} \mathcal{O}$ 
which vanish on $V^{\prime}$, because $P_{j}$ is also the minimal analytic polynomial of $z_{j}$ on $V^{\prime}$ by construction.

Proposition 3.5 (Analytic polynomial). Under the same bypothesis and notation as Proposition 3.4, for every $f \in \Theta(\Omega)$, there is a unique monic pseudopolynomial $P\left(z^{\prime}, t\right) \in \mathcal{O}\left(\Omega^{\prime}\right)[t]$ of degree $k$ equal to the sheeting order of $\pi$ such that $\left\{t \in \mathbf{C} \mid P\left(z^{\prime}, t\right)=0\right\}=f\left(\pi^{-1}\left(z^{\prime}\right)\right)$.

Proof. Let $P\left(z^{\prime}, t\right)=\Pi_{i=1}^{k}\left(t-f\left(w_{i}\left(z^{\prime}\right)\right)\right)$ and the required properties follow as before. The polynomial of this proposition will be called the analytic polynomial and that of Proposition 3.4 will be called the minimal analytic polynomial. In the event that $f$ separates the fibers of $\pi$, the se polynomials will concur.

Definition. A complex linear function $L(z)=a_{1} z_{1}+\cdots+a_{n} z_{n}$ is said to be a regular direction if $L(z)$ separates the fibers $\pi^{-1}\left(z^{\prime}\right)$ over an open dense set of points, i.e., the values $L w_{1}\left(z^{\prime}\right), \cdots, L w_{k}\left(z^{\prime}\right)$ are distinct for all $z^{\prime}$ in an open dense subset of $\Omega^{\prime}$. It is clear that there are lots of these; in fact, representing $L$ by the element $a=\left[a_{1}, \cdots, a_{n}\right]$ of projective space, we have

Proposition 3.6. Let $W=V \times G(n-r, n)-B$; then $Q=\{(p, T, a) \epsilon$ $V \times G(n-r, n) \times \mathbf{C P}^{n-1} \mid T$ good at $p, a \subset T$, but a not a regular direction $\}$ is an analytic set in $W \times \mathbf{C} \mathbf{P}^{n-1}$.

To prove this we first need $\mathrm{CP}^{n-1}$.

Lemma 3.7. $\left\{(T, a) \in G(k, n) \times \mathbf{C P}^{n-1} \mid a \subset T\right\}$ is an analytic set in $G(k, n) \times$

Proof. Let $T$ be represented by $k \times n$ matrix with row vectors $r_{1}, \cdots, r_{k}$. There is a holomorphic embedding $G(k, n) \rightarrow \mathbf{C} \mathbf{P}^{N-1}, N=\left(\begin{array}{l}n \\ k\end{array}\right)$, given by $\left(r_{1}, \cdots\right.$, $\left.r_{k}\right) \rightarrow r_{1} \wedge r_{2} \wedge \ldots \wedge r_{k}$, where $C^{N}$ is identified with $\wedge^{k} C^{n}$. Then $a \subset T$ if and only if $a \wedge r_{1} \wedge \ldots \wedge_{r_{k}}=0$.

Proof of Proposition 3.6. Let $\pi: W \rightarrow \mathbf{C}^{r} \times G(n-r, n)$ be the holomorphic map defined by $\pi(p, T)=\left(\pi_{T}(p), T\right)$. The fibers of $\pi, \pi^{-1} \pi(p, T)=V \cap(T+p) \times T$ are discrete so $\pi$ is locally an analytic cover. For each $(p, T) \in W$, there is a neighborhood $U$ of $(p, T)$ in $W$ and a proper subvariety $Z$ of the open set $\pi(U)$ such that $\pi \mid U$ is proper and $\pi: U-\pi^{-1}(z) \rightarrow \pi(U)-Z$ is a $k$-sheeted covering map. For $\left(p^{\prime}, T^{\prime}\right) \notin Z$, there are holomorphic functions $w_{1}\left(p^{\prime}, T^{\prime}\right), \cdots, w_{k}\left(p^{\prime}, T^{\prime}\right)$ whose values lie in $\mathrm{C}^{n}$ and are the points of the set $V \cap\left(T^{\prime}+p^{\prime}\right)$.

It is necessary to know that for no $T$ do we have $\mathrm{C}^{r} \times T \subset Z$. To see this, we must explicitly define $Z=\pi(\operatorname{Sing} U \cup\{$ Regular points of $U$ where the Jacobian rank of $\pi<r+r(n-r)\})$. 
Now Sing $U \subset \operatorname{Sing} V \times G(n-r, n)$ and for regular points $(p, T) \in U, \operatorname{rank}_{(p, T)^{\pi}=}$ $\operatorname{rank}_{p} \pi_{T}+r(n-r)$ so

$\left(C^{r} \times T\right) \cap Z \subset \pi_{T}\left(\operatorname{Sing}(V) \cup\left\{\right.\right.$ Regular points of $V$ where rank $\left.\left.\pi_{T}<r\right\}\right) \times T$.

This latter set has $\operatorname{dim} \leq r-1$, so $\mathbf{C}^{r} \times T \not \subset Z$.

Now if $a \subset T$ and $a$ separates the fiber $\pi_{T}^{-1}\left(p^{\prime}\right)$, then $a$ separates all fibers $\pi_{T}^{-1}\left(z^{\prime}\right)$ such that $\delta\left(z^{\prime}\right) \neq 0$, where $\delta$ is the discriminant of the minimal analytic polynomial for the linear function associated to $a$. Thus we have the following facts:

(1) If $(p, T, a)$ is regular, then $\left(p^{\prime}, T, a\right)$ is regular for all $\left(p^{\prime}, T\right) \in U$.

(2) If $(p, T) \notin Z, a \subset T$, and $a$ separates $V \cap(T+p)$, then $(p, T, a)$ is regular.

(3) If $(p, T, a)$ is regular, then a separates $V \cap\left(T+p^{\prime}\right)$ for some $\left(p^{\prime}, T\right) \notin Z$.

It now follows that $(p, T, a) \in Q$ if and only if $a \subset T, T$ is good at $p$, and $a \cdot\left(w_{i}\left(p^{\prime}, T\right)-w_{j}\left(p^{\prime}, T\right)\right)=0$ for all $\left(p^{\prime}, T^{\prime}\right) \notin Z$ and some $i \neq j$.

Lemma 3.8. Let $P\left(z^{\prime}, t\right)$ be the minimal analytic polynomial for $z_{j}$ in $\Theta\left(\Omega^{\prime}\right)[t] \cap I(V)$; then

(a) $\left(\partial^{i} P / \partial t^{i}\right)\left(x^{\prime}, x_{j}\right)=0$ for $i=0, \cdots, \lambda-1 \Rightarrow$ the sheeting order of $\pi$ at $\left(x^{\prime}, x_{j}\right)$ is $\geq \lambda$;

(b) if $z_{j}$ is a regular direction and the sheeting order of $\pi$ at $\left(x^{\prime}, x_{j}\right)$ is $\geq \lambda$, then $\left(\partial^{i} P / \partial t^{i}\right)\left(x^{\prime}, x_{j}\right)=0$ for $i=0, \cdots, \lambda-1$.

Proof. (See Whitney [16, Theorem VII-8E].)

(a) $P\left(z^{\prime}, z_{j}\right)=\left(z_{j}-x_{j}\right)^{k}+b_{1}\left(z^{\prime}\right)\left(z_{j}-x_{j}\right)^{k-1}+\cdots+b_{k}\left(z^{\prime}\right), \partial^{i} P\left(x^{\prime}, x_{j}\right) / \partial t^{i}=$ $i ! b_{k-i}\left(x^{\prime}\right)$ so $b_{k}\left(x^{\prime}\right)=\cdots=b_{k-\lambda+1}\left(x^{\prime}\right)=0$. $P\left(x^{\prime}, z_{j}\right)=\left(z_{j}-x_{j}\right)^{\lambda} Q\left(x^{\prime}, z_{j}\right)$.

$P$ has a zero of order $\lambda$ at $\left(x^{\prime}, x_{j}\right)$ so by continuity of the roots, for $z^{\prime}$ near $x^{\prime}, P$ has $\lambda$ roots near $x_{j}$. But since $P$ is minimal, each root arises from a point of the fiber $\pi^{-1}\left(z^{\prime}\right)$.

(b) There are at least $\lambda$ distinct values $r_{i}=z_{j}\left(w_{i}\left(z^{\prime}\right)\right)$ near $x_{j}$ which are roots of $P\left(z^{\prime}, z_{j}\right)$ for most $z^{\prime}$ near $x^{\prime}$.

$$
\begin{aligned}
P\left(z^{\prime}, z_{j}\right) & =\prod_{i=1}^{k}\left(z_{j}-z_{j} w_{i}\left(z^{\prime}\right)\right), \\
\frac{\partial^{m} P}{\partial t^{m}}\left(z^{\prime}, z_{j}\right) & =\sum_{\substack{H \subset\{1, \cdots, k\} \\
H(H)=k-m}} \prod_{i \in H}\left(z_{j}-z_{j} w_{i}\left(z^{\prime}\right)\right) .
\end{aligned}
$$

For $m \leq \lambda-1$, each term of the above sum has some $r_{i}$ in the product, so as $\left(z^{\prime}, z_{j}\right) \rightarrow\left(x^{\prime}, x_{j}\right)$ each term of sum $\rightarrow 0$. 
Lemma 3.9. Let $P\left(z^{\prime}, t\right)$ be the analytic polynomial for $z_{j}$ in $\Theta\left(\Omega^{\prime}\right)[t] \cap I(V)$; then $\left(\partial^{i} P / \partial t^{i}\right)\left(x^{\prime}, x_{j}\right)=0$ for $i=0, \cdots, \lambda-1$ if and only if the sheeting order of $\pi$ at $\left(x^{\prime}, x_{j}\right)$ is $\geq \lambda$. (Proof is similar to last lemma.)

Theorem 3.1 will be proved by first showing that $M_{k}-B=\{(p, T) \in V \times G-$ $B \mid \mu(V, p, T) \geq k\}$ is analytic in $V \times G-B$, explicitly computing the equations for $B$ to see that this set extends across $B$. The special case of a hypersurface will be considered first to simplify matters.

We want to express the condition of Lemma 3.5 invariantly of the direction $z_{n}$. If $V$ is a hypersurface, then $z_{n}$ is surely a regular direction. For any $f \in I(V)$, there exists $g \in \mathcal{O}(\Omega)$ so that $f(z)=g(z) P\left(z^{\prime}, z_{n}\right)$. Hence by Leibnitz's rule, $\pi$ has sheeting order $\geq \lambda$ at $z$ if and only if $\left(\partial^{i} f / \partial z_{n}^{i}\right)(z)=0$ for $i=0, \cdots$, $\lambda-1$. Let $f(z)=f(p)+\Sigma_{j=1}^{\infty} \Sigma_{|a|=j} f_{a}(p) z^{\alpha}$ be the power series expansion of $f$ about $p$; the $j$ th directional derivative of $f$ at $p$ in the direction $a=\left(a_{1}, \cdots, a_{n}\right)$ is $\Sigma_{|a|=j} f_{\alpha}(p) a^{\alpha}$. Thus $\mu(V, p, a) \geq k \Leftrightarrow \Sigma_{|a|=j} f_{\alpha}(p)^{\alpha}=0$ for $j=0, \cdots, k-1$ and all $f \in I(V)$. But $a$ is bad for $V$ at $p$ if and only if the same equations hold for all $j$ and all $f \in I(V)$. Thus $M_{k}$ is analytic in $V \times G$.

To handle the nonhypersurface case, consider the holomorphic map:

$$
\begin{gathered}
\left\{(T, a) \in G(n-r, n) \times \mathrm{CP}^{n-1} \mid a \subset T\right\} \rightarrow G^{\prime}(n-r-1, n-1), \\
(T, a) \rightarrow T^{\prime}
\end{gathered}
$$

such that $T^{\prime} \oplus a=T$, where $G^{\prime}(n-r-1, n-1)$ is the subset of elements of $G(n-r, n)$ of the form $\left[I_{n-r-1}, 0_{(n-r-1) \times 1},{ }_{(n-r-1) \times r}\right]$ (see Theorem 2.3). Consider the following diagram of holomorphic maps:

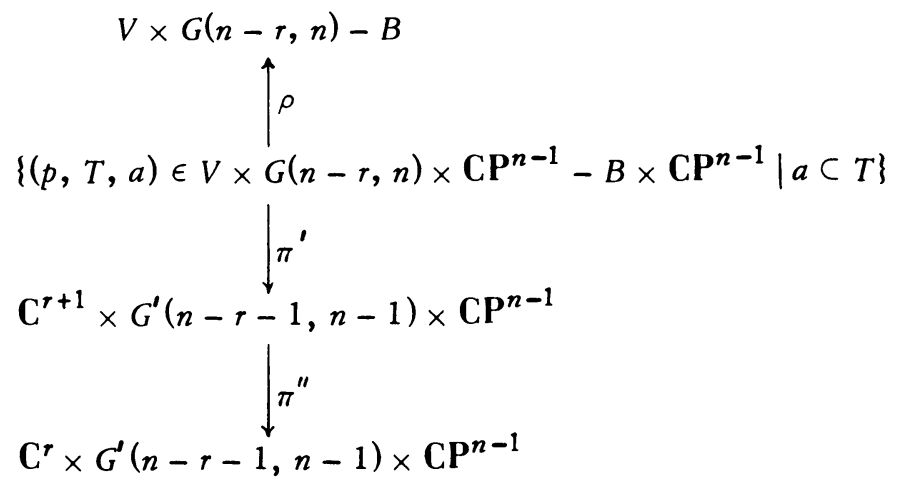

where $\rho(p, T, a)=(p, T)$ is proper, $\pi^{\prime}(p, T, a)=\left(\pi_{T},(p), T^{\prime}, a\right), \pi^{\prime \prime}\left(q, T^{\prime}, a\right)=$ $\left(\pi_{a}(q), T^{\prime}, a\right)$ where $\pi_{T}$, is projection along $T^{\prime}$ to $\mathbf{C}^{r+1}=\operatorname{span}$ of $\mathbf{C}^{r}$ and $a$. Now 
$\pi^{\prime}$ has discrete fibers and locally its image is an analytic set of pure codimension one and so is the locus of $f\left(z_{1}, \cdots, z_{r+1}, T^{\prime} a\right)$ (the domain is a complex manifold). In fact, through use of the local parametrization $\pi^{\prime \prime}, f$ can be chosen to be a pseudopolynomial in $z_{r+1}$ so that for each fixed $T^{\prime}$ and $a, f\left(z, T^{\prime}, a\right)$ vanishes on $\pi_{T^{\prime}}(V)$ but does not necessarily generate $I\left(\pi_{T^{\prime}}(V)\right)=I(V) \cap_{r+1} \mathcal{O}$. Now $\pi^{\prime \prime}$ is locally an analytic cover with branches $w_{1}\left(z^{\prime}, T^{\prime} a\right), \cdots, w_{m}\left(z^{\prime}, T^{\prime}, a\right)$ and by the construction used in Proposition 3.6, for all $T^{\prime}$ and $a, w_{1}\left(z^{\prime}\right), \cdots, w_{m}\left(z^{\prime}\right)$ are just the distinct branches of $\pi_{T}: V \rightarrow \mathrm{C}^{r}$. However as $z^{\prime} \rightarrow x^{\prime}$, all the branches $w_{i}\left(z^{\prime}\right)$ might not converge to the same value. Let

$$
f\left(z, T^{\prime}, a\right)=\prod_{i=1}^{m}\left(z_{r+1}-z_{r+1}\left(w_{i}\left(\pi^{\prime \prime}(z)\right)\right)\right) .
$$

Let $M_{k}^{\prime}=\left\{\left(q, T^{\prime}, a\right) \mid \Sigma_{|a|=j} f_{a}\left(q, T^{\prime}, a\right) a^{\alpha}=0\right.$ for all $a \subset T$ and $j=0, \cdots$, $k-1\}$; then as in Lemmas 4.8 and $4.9, M_{k}=\rho\left(\pi^{\prime}\right)^{-1}\left(M_{k}^{\prime}\right)$ so $M_{k}$ is analytic in $V \times G-B$. Furthermore $(p, T) \in M_{k}-B$ if and only if $0=\Sigma|a|=j f_{\alpha}(p) a^{\alpha}$ for $j=0, \cdots, k-1$, all $a \subset T$ and a specific $f \in I(V) \cap_{r+1} \mathcal{O}$. Comparing with $\$ 2.2$, which says that $(p, T) \in B$ if and only if the se equations are satisfied for all $j$, all $a \subset T$, and all $f \in I(V) \cap_{r+1} \mathcal{O}$, we see that $M_{k}$ extends across $B$.

3.2. To each point $p \in V$, there are associated a minimal and maximal multiplicity, denoted $\mu(V, p)$ and $m(V, p)$ respectively, where $\mu(V, p)=$ $\min T_{\text {good for } V \text { at } p} \mu(V, p, T)$. We recover the theorem of Whitney [16, VII, $8 A+$ $8 \mathrm{E}]$.

Corollary 3.10. $\{p \in V \mid \mu(V, p) \geq k\}=\bigcap_{T \in G(n-r, n)}\{p \in V \mid \mu(V, p, T) \geq k\}$ is an analytic set in $V$.

The minimal multiplicity of a point is invariant under biholomorphic mappings but the maximal multiplicity is not invariant; $\mu(V, p)=1$ if and only if $p$ is a regular point of $V$; for $p \in \operatorname{Reg}(V), \mu(V, p, T)=1$ if and only if $\pi_{T}$ is a biholomorphism on the germ of $V$ at $p$.

I do not know if $\{p \in V \mid m(V, p) \geq k\}$ is an analytic set unless $k=2$, in which case it is analytic.

Lemma 3.11. $m(V, p)=1$ if and only if $V$ is a complex linear subspace of $\mathrm{C}^{n}$ near $p$.

Proof. Suppose $m(V, p)=1$; then $p$ is a regular point and let $T_{p} V$ denote the tangent space to $V$ at $p$. If $V \neq T_{p} V+p$ near $p$, let $L$ be a complex line contained in $T_{p} V$ such that $\operatorname{dim}_{p}(V \cap(L+p))=0$. There exists a $n-r \operatorname{dim}$ plane $T^{\prime}$ containing $L$ such that $\operatorname{dim}_{p}\left(V \cap\left(T^{\prime}+p\right)\right)=0$. Hence $\pi_{T}$, kills $L$ and is not a biholomorphism on $V$ near $p$ so $m(V, p) \geq \mu\left(V, p, T^{\prime}\right)>1$, a contradiction.

It follows that $\{p \in V \mid m(V, p)=1\}$ is just the union of the irreducible 
components of $V$ which are linear subspaces of $V$ and $\{p \in V \mid m(V, p) \geq 2\}$ is the union of the remaining components.

It is easy to compute the multiplicities for certain special cases which show that there is very little relationship between the minimal and maximal multiplicity.

Let $V_{n}=\left\{\left(z_{1}, z_{2}, z_{3}\right) \in \mathrm{C}^{3} \mid z_{2}^{q}{ }^{n}=z_{1}^{p}{ }^{n}\right.$ and $\left.z_{3}=n\right\}$ and $V=\bigcup_{n=1}^{\infty} V_{n}$, where $p_{n}$ and $q_{n}$ are relatively prime integers. Then

$$
\begin{aligned}
\mu\left(V,\left(z^{q} n, z^{p_{n}}, n\right),\left(q_{n} z^{q_{n}-1}, p_{n} z^{p_{n}-1}, 0\right)\right) & =m\left(V,\left(z^{q}, z^{p_{n}}, n\right)\right) \\
& = \begin{cases}\max \left(p_{n}, q_{n}\right), & z=0 \\
2, & z \neq 0\end{cases}
\end{aligned}
$$

and

$$
\mu\left(V,\left(z^{q}{ }^{n}, z^{p} n, n\right),(0,1,0)\right)=\mu\left(V,\left(z^{q}{ }^{n}, z^{p}, n\right)\right)= \begin{cases}\min \left(p_{n}, q_{n}\right), & z=0 \\ 1 & z \neq 0\end{cases}
$$

Thus by picking each $p_{n}=1$ and $q_{n} \rightarrow \infty$, we have an example with the minimal multiplicity bounded by 1 and the maximal multiplicity unbounded on a variety.

There is also such an example with $V$ irreducible. Let $\left\{a_{n}\right\}$ be any discrete sequence of points in the complex plane $\mathrm{C}$; by the Weierstrass theorem, there is an entire function $f$ with zeros of order $p_{n}-1$ at the points $a_{n}$. Let $g$ be an entire function whose derivative is $f$ and let $A$ be the image of the function: $\mathrm{C} \rightarrow \mathrm{C}^{2}$ given by $t \rightarrow(t, g(t))$; then $A$ is a complex manifold so the minimal multiplicity is indentically one on $A$. Near the points $\left(a_{n}, g\left(a_{n}\right)\right), A$ looks like $z_{1}^{p}{ }^{n}=z_{2}$ so has maximal multiplicity $p_{n}$.

Proposition 3.12. The maximal multiplicity is bounded on an algebraic variety; more precisely if $V$ is an algebraic subvariety of $\mathbf{C}^{n}$ of pure dimr, then there exists an integer $K>0$ such that $\mu(V, p) \leq K$ for all $p \in V$.

Proof. First consider the hypersurface case-let $V$ be an algebraic subvariety of pure codim one. Then $V$ can be expressed globally as an analytic cover over $\mathrm{C}^{n-1}$. Let $P\left(z^{\prime}, z_{n}\right)$ be the analytic polynomial for $z_{n}$; the coefficients of $P$ are entire functions with polynomial growth and so are polynomials (see Rudin [13, Theorems 1 and 2]). Let $\operatorname{deg} P$ denote the homogeneous degree of $P$. It follows that $m(V, p) \leq \operatorname{deg} P$ for all $p \in V$ since: $a$ bad at $p \in V \Leftrightarrow a$ satisfies all the homogenous polynomials $\Sigma_{|a|=j} f_{a}(p) a^{a}$ for all $f \in I(V) ; \mu(V, p, a) \geq k \Leftrightarrow a$ satisfies the first $k-1$ homogenous polynomials for all $f \in I(V) \Leftrightarrow a$ satisfies the 
first $k-1$ homogeneous polynomials of $P$, since $P$ generates $I(V)$.

More generally, a careful examination of the proof of Theorem 4.1 shows that $M_{k}$ is an algebraic subvariety of $\mathbf{C}^{n} \times G(n-r, n): \pi^{\prime}\left(V \times G(n-r, n) \times \mathbf{C P}^{n-1}-B \times \mathbf{C P} n-1\right)$ is contained in an algebraic subvariety of pure codim one. By use of the parametrization $\pi^{\prime \prime}, f\left(z_{1}, \cdots, z_{r+1}, T^{\prime}, a\right)$, the analytic polynomial of $z_{r+1}$ is actually a polynomial and $M_{k}=\left\{(p, t) \mid \Sigma_{|a|=j} f_{a}\left(p, T^{\prime}, a\right) a^{a}=0\right.$ for all $a \subset T$ and $j=0, \cdots, k-1\}$. Thus $m(V, p) \leq \operatorname{deg} f$.

Remark. It is well known that the polynomial ring $\mathrm{C}\left[z_{1}, \cdots, z_{n}\right]$ is Noetherian, so the ideal of all polynomials vanishing on $V$ is generated by finitely many elements $P_{1}, \cdots, P_{m}$. It follows that $P_{1}, \cdots, P_{m}$ also generate the ideal of all holomorphic functions vanishing on $V$ [13, Theorems 4.1, 4.4, and 5]. However if $V$ is not an algebraic variety, this construction fails and it is not necessarily true that the ideal of all holomorphic functions vanishing on $V$ is finitely generated.

3.3. Let $\mathcal{C}_{p}(V)$ be the Whitney tangent cone to $V$ at $p$ ([14], [15]); $a \in \mathcal{C}_{p}(V)$ if and only if there exist $q_{i} \in V$ with $q_{i}-p$ converging to $a$ in $\mathbf{C P}^{n-1}$. Equivalently $\mathcal{C}_{p}(V)$ is the common locus of the initial polynomials of all holomorphic functions vanishing on $V$ near $p . \mathcal{C}_{p}(V)$ is an analytic variety of the same dimension as $V$ and $\mu(V, p, T)=\mu(V, p)$ if and only if $\operatorname{dim}\left(\mathcal{C}_{p}(V) \cap T\right)=0$.

Let $P\left(z^{\prime}, z_{j}\right)$ be the minimal analytic polynomial for $z_{j}$; then $\mu(V, p, T) \geq$ degree of $P$ in $z_{j} \geq$ order of $P$.

Proposition 3.13. If $z_{j}$ is a regular direction, then $\operatorname{dim}\left(\mathcal{C}_{p}(V) \cap T\right) \leq 1 \Leftrightarrow$ ord $P=\mu(V, p)$.

Proof. First consider the hypersurface case, where the first condition is automatically satisfied since $\operatorname{dim} T=1 ; P$ generates the ideal $I(V)$ and the order of products is the sum of orders, so $P$ has minimal order with respect to elements of $I(V)$. Choosing a direction $T \in \mathbf{C P}^{n-1}$ with $\mu(V, p, T)=\mu(V, p)$, we see that ord $P=\mu(V, p)$.

More generally, $T$ can be written as $T^{\prime} \oplus L$, where $T$ is a $n-r-1$ dim plane and $L$ is the complex line determined by $z_{j}$. Now the linear function $z_{j}$ and hence $P\left(z^{\prime}, z_{j}\right)$ do not depend upon the choice of $T^{\prime}$, so pick $T^{\prime}$ so that $\operatorname{dim}\left(\mathcal{C}_{p}(V) \cap T^{\prime}\right)=0$. Let $\mathbf{C}^{r+1}=\mathbf{C}^{r} \oplus L, V^{\prime}$ be the locus in $\mathbf{C}^{r+1}$ of $P, D$ be the locus in $C^{r}$ of the discriminant of $P$, and $D^{\prime}=\pi_{L}^{-1}(D)$. Then $P_{j}$ is also the minimal analytic polynomial of $V^{\prime}, \pi_{T}(V)=V^{\prime}$, and $\pi_{T^{\prime}}: V-\pi_{T^{\prime}}^{-1}\left(D^{\prime}\right) \rightarrow V^{\prime}-D^{\prime}$ is one-to-one since $z_{j}$ is a regular direction. For any local parametrization $\pi_{a}: V^{\prime} \rightarrow \mathbf{C}^{r}, a \in \mathbf{C P}^{n^{-1}}: \mu\left(V^{\prime}, p, a\right)=\left(\#\right.$ sheets of $\left.\pi_{T}{ }^{\prime}\right) \cdot\left(\#\right.$ sheets of $\left.\pi_{a}\right)=$ $\mu\left(V, p, T^{\prime} \oplus a\right) \geq \mu(V, p)$. 
Hence by the above, we have ord $P=\mu\left(V^{\prime}, p\right) \leq \mu\left(V^{\prime}, p, a\right)=$ $\mu\left(V, p, T^{\prime} \oplus a\right) \geq \mu(V, p)$. If $a$ is chosen so that $a \notin \mathcal{C}_{p}\left(V^{\prime}\right)=\pi_{T}{ }^{\prime}\left(\mathcal{C}_{p}(V)\right)$, then $\operatorname{dim}\left(\mathcal{C}_{p}(V) \cap\left(T^{\prime} \oplus a\right)\right)=0$, so ord $P=\mu(V, p)$.

4. Finitely generated varieties. As remarked earlier, the ideal of a variety is not necessarily finitely generated. In this section, I give an example of a variety that is not finitely generated and a geometric condition which implies the ideal is finitely generated.

Let $V$ be an analytic subvariety of pure $\operatorname{dim} r$ of an open subset $\Omega$ of $\mathbf{C}^{n}$. Denote by $I(V, p)$ the ideal in ${ }_{n} \mathcal{O}_{p}$ of all functions vanishing on the germ of $V$ at $p$ and by $I(V)$ the ideal in $Q(\Omega)$ of functions vanishing on $V$. Let $\# I(V, p)$ denote the minimal number of generators of $I(V, p)$ over ${ }_{n}{ }_{p}$, which is unique by Nakayama's lemma since ${ }_{n} \mathcal{O}_{p}$ is a local Noetherian ring.

Theorem 4.1. \#I(V,p) $\leq[\mu(V, p)+1]^{n}$. If $r=1$ or 2 , \#I(V,p) $2(n-r)[\mu(V, p)+1]^{n-r}$.

This local result can be combined with the following theorem of Kripke [9, Theorem 1] to get global results $-I(V)$ is finitely generated if the minimal multiplicity of $V$ is bounded.

Theorem. Let $X$ be an $r$-dim analytic space and $\Im$ a coberent analytic sheaf on $X$ such that the global sections $\Im_{(X)}$ generate each stalk $\Im_{x}$. If \# $\Im_{x} \leq k$ for every $x \in X$, then $\left\{\left(s_{1}, \cdots, s_{k(r+1)}\right) \in \mathfrak{\Im}^{k(r+1)}(X) \mid s_{1}, \cdots, s_{k(r+1)}\right.$ generate $\Im(X)$ \} is the complement of a first category set in $\Im^{k(r+1)}(X)$.

Corollary 4.2. Also assume that $\Omega$ is a domain of bolomorpby. If $\mu(V, p)<K$ for all $p \in V$, then $\# I(V) \leq(r+1) K^{n}$. If $r=1$ or $2, \# I(V) \leq 2(r+1)(n-r) K^{n-r}$.

Remark. In the above corollary, the assumption about $\Omega$ can be weakenedinstead of $\Omega$ being a domain of holomorphy, it is enough to have $V=$ $\bigcap_{f \in I(V)}$ locus $(f)$ because it then follows that there are enough global functions to generate each stalk $I(V, p)$ of the coherent sheaf $g(V)$. Let $\widetilde{\Omega}$ be the envelope of holomorphy of $\Omega$ and $\tilde{V}$ be the common locus of all $f \in I(V) \subset \mathcal{O}(\Omega)=\mathcal{O}(\tilde{\Omega})$. Then $\widetilde{\Omega}$ is a Stein manifold so by Cartan's Theorem $A, I(V)$ generates $I(\widetilde{V}, p)$ for all $p \in \tilde{V}$.

The proof of Theorem 4.1 is basically a big chase through the proof of Oka's lemma-coherence of $\mathcal{O}$. The ideal $I(V, p)$ can be viewed as the sheaf of relations between some special functions and the proof of Oka's lemma as an algorithm for computing the generators. The bound on the multiplicity enables one to put a bound on the number of resulting generators.

Let $p \in V$, and $\pi: \mathbf{C}^{n} \rightarrow \mathbf{C}^{r}$ be a projection expressing $V$ in a neighborhood 
of $p$ as an analytic cover of minimal sheeting order $k=\mu(V, p)$. Since for $T \in G(n-r, n)$ near $\mathrm{C}^{n-r}, \mu(V, p, T)=\mu\left(V, p, \mathrm{C}^{n-r}\right)=k$, we can also assume that $\pi$ has no branches which are identically zero. Also choose $\pi$ so that $\mathbf{C}^{r}$ is not contained in the tangent cone to $V$ at $p$, e.g., $\operatorname{dim}\left(\mathbf{C}^{r} \cap \mathcal{C}_{p}(V)\right) \leq r-1$. Now $\pi$ is also a parametrization of $\mathcal{C}_{p}(V)$ and one can apply the usual the ory of analytic covers to it as well as $V$.

Choose a basis of $\mathrm{C}^{n-r}$ so that $z_{r+1}, \cdots, z_{n}$ are all regular directions for both $\pi: V \rightarrow \mathrm{C}^{r}$ and $\pi: \mathcal{C}_{p}(V) \rightarrow \mathrm{C}^{r}$; this can be done since the set of nonregular directions forms a linear subvariety of $\mathbf{C P}^{n-1}$ by Proposition 3.6. It is also possible to choose this basis so that no $z_{j}, r+1 \leq j \leq n$, vanishes identically on any branch of $\pi$ on $V$ or $\mathcal{C}_{p}(V)$. Let $P_{j}\left(z^{\prime}, z_{j}\right)=z_{j}^{k}+a_{1 j}\left(z^{\prime}\right) z_{j}^{k-1}+\cdots+a_{k j}\left(z^{\prime}\right)$ be the analytic polynomial for $z_{j}$ of $V$. Then the above conditions insure that $a_{k j}\left(z^{\prime}\right) \equiv 0$, since $a_{k j}$ is the product of the values of $z_{j}$ on the branches of $\pi$.

Lemma 4.3. There exists a bas is of $\mathrm{C}^{r}$ so that the tail coefficients $a_{k j}\left(z^{\prime}\right)$ vanish to order no more than $\mu(V, p)$ in each direction $z_{1}, \cdots, z_{r}$.

Proof. Let $V^{\prime}$ be the locus of $P_{j}$ in $\mathrm{C}^{r+1}=\operatorname{span}$ of $\mathrm{C}^{r}$ and $z_{j}$, and let $T^{\prime}$ be a $n-r-1$ dim plane in $\mathrm{C}^{n-r}$ such that $\mathrm{C}^{n}=\mathrm{C}^{r+1} \oplus T^{\prime}$. For any direction $L=c_{1} z_{1}+\cdots+c_{r+1} z_{r+1}$ in $\mathrm{C}^{r+1}$ which is good for $V^{\prime}, \mu\left(V^{\prime}, p, L\right)=$ $\mu\left(V, p, T^{\prime} \oplus L\right) \leq m(V, p)$. For any direction $L$ in $\mathbf{C}^{r}, \mu\left(V^{\prime}, p, L\right) \geq l \Leftrightarrow P_{j}$ vanishes to order $\geq l$ in the direction $L \Leftrightarrow a_{k j}\left(z^{\prime}\right)$ vanishes to order $\geq l$ in the direction $L$. Now $a_{k j} \equiv 0$, so $a_{k j}$ vanishes to order $\leq m(V, p)$ in any direction which is a nonsolution to the initial polynomial of $a_{k}$.

But it is also possible to choose direction $L$ in $\mathrm{C}^{r}$ so that $\operatorname{dim}\left(\mathcal{C}_{p}(V) \cap\left(T^{\prime} \oplus L\right)\right)=0$; then $\mu\left(V^{\prime}, p, L\right)=\mu(V, p)$ and so $a_{k j}$ vanishes to order only $\mu(V, p)$ in the direction $L$. Such directions are constructed as follows: Now $\operatorname{dim}\left(T^{\prime} \cap \mathcal{C}_{p}(V)\right)=0$ so $\left.\pi_{T}, \mathcal{C}_{p}(V)\right)$ is analytic in $\mathbf{C}^{r+1}$ and of pure $\operatorname{dim} r$. Also $\operatorname{dim}\left(\mathbf{C}^{r} \cap \pi_{T},\left(\mathcal{C}_{p}(V)\right)\right) \leq r-1$ because $z_{j}$ vanishes identically on no branch of $\pi$ on $\mathcal{C}_{p}(V)$. Since $\operatorname{dim}\left(\mathcal{C}_{p}(V) \cap\left(T^{\prime} \oplus L\right)\right)=0$ is equivalent to $\operatorname{dim}\left(L \cap \pi_{T^{\prime}}\left(C_{p}(V)\right)\right)=0$, there are lots of such directions.

This basis of $\mathbf{C}^{r}$ can also be chosen to satisfy the additional condition that for any $a_{i j} \not \equiv 0, a_{i j}$ does not vanish identically in any of the directions $z_{1}, \cdots$, $z_{r}$, by choosing directions which are nonsolutions to the respective initial polynomials.

Later on it will be necessary to write each $a_{i j}\left(z^{\prime}\right)$ as a unit times a Weierstrass polynomial in $z_{r}$ with coefficients in ${ }_{r-1} \Theta_{p}$, and then write each resulting coefficient again as a unit times a Weierstrass polynomial in $z_{r-1}$ with coefficients in ${ }_{r-2} \Theta_{p}$, etc. The above conditions on the basis of $\mathbf{C}^{r}$ guarantee that this 
is possible and that for a tail coefficient

$$
\begin{aligned}
a_{k j}\left(z_{1}, \cdots, z_{r}\right)= & u\left(z_{1}, \cdots, z_{r}\right) \\
& \cdot\left[z_{r}^{l}+a_{1 k j}\left(z_{1}, \cdots, z_{r-1}\right) z_{r}^{l-1}+\cdots+a_{l k j}\left(z_{1}, \cdots, z_{r-1}\right)\right],
\end{aligned}
$$

$l \leq \mu(V, p)$ and again $a_{l k j}$ vanishes to order $\leq \mu(V, p)$ in the directions $z_{1}, \cdots, z_{r-1}$.

Now with the fixed basis of $C^{n}$ determined above, we proceed to construct some canonical functions of the variety.

Lemma 4.4. For every $f \in{ }_{n} \mathcal{O}_{p}$, there is a unique pseudopolynomial $Q_{f} \in{ }_{r} \mathcal{O}_{p}[t]$ of degree $\leq k-1$ such that $f(z) \delta(\pi(z))-Q_{f}\left(\pi(z), z_{r+1}\right) \in I(V, p)$, where $\delta$ is the discriminant of the polynomial $P_{r+1}\left(z^{\prime}, z_{r+1}\right)$. In particular, there are $Q_{j} \in \underset{r}{\mathcal{O}_{p}}\left[z_{j}\right]$ for $j=r+2, \cdots$, n so that $z_{j} \delta\left(z^{\prime}\right)-Q_{j}\left(z^{\prime}, z_{r+1}\right) \in I(V, p)$.

This is proved by algebraic methods in [11, Lemma 2, p. 35] for irreducible varieties and by geometric means in $[6$, Theorem 18, p. 113] for pure dim varieties as follows:

$$
\delta\left(z^{\prime}\right)=\prod_{i \neq j}\left(z_{r+1}\left(w_{i}\left(z^{\prime}\right)\right)-z_{r+1}\left(w_{j}\left(z^{\prime}\right)\right)\right) \not \equiv 0
$$

as $z_{r+1}$ is a regular direction, where the $w_{i}$ are the branches of $\pi$. Now for any $f \in \underset{n}{\mathcal{O}}$, we want to find $b_{j} \in \underset{r}{\mathcal{O}}$ such that

$$
\delta\left(z^{\prime}\right) f\left(w_{i}\left(z^{\prime}\right)\right)=\sum_{j=0}^{k-1} b_{j}\left(z^{\prime}\right) z_{r+1}\left(w_{i}\left(z^{\prime}\right)\right)^{j} \quad \text { for } i=1, \ldots, k
$$

These equations can be viewed as a system of $k$ linear equations in the $k$ unknown values $b_{j}\left(z^{\prime}\right)$; hence by Cramer's rule

$$
\begin{aligned}
& b_{j}\left(z^{\prime}\right) \operatorname{det}\left[1, z_{r+1} w_{i}\left(z^{\prime}\right), \ldots, z_{r+1} w_{i}\left(z^{\prime}\right)^{k-1}\right] \\
& =\operatorname{det}\left[1, z_{r+1} w_{i}\left(z^{\prime}\right), \ldots, z_{r+1} w_{i}\left(z^{\prime}\right)^{j-1}, .\right. \\
& \left.\quad \delta\left(z^{\prime}\right) f w_{i}\left(z^{\prime}\right), z_{r+1} w_{i}\left(z^{\prime}\right)^{j+1}, \ldots, z_{r+1} w_{i}\left(z^{\prime}\right)^{k-1}\right] .
\end{aligned}
$$

The determinant appearing in the left-hand side is the van der Monde determinant $\Delta$, and it is well known that $\Delta^{2}=\delta$. Factoring $\delta$ out of the right-hand side of the equations produces the explicit formula

$$
b_{j}=\Delta \cdot \operatorname{det}\left[1, z_{r+1}\left(w_{i}\right), \ldots, z_{r+1}\left(w_{i}\right)^{j-1}, f\left(w_{i}\right), z_{r+1}\left(w_{i}\right)^{j+1}, \ldots, z_{r+1}\left(w_{i}\right)^{k-1}\right] .
$$


Lemma 4.5. For $N \geq(k-1)(n-r-1), f \in I(V, p)$ if and only if $\delta^{N} f$ lies in the ideal of ${ }_{n} \mathcal{O}_{p}$ generated by $P_{r+1}, \cdots, P_{n}, z_{r+2} \delta-Q_{r+2}, \cdots, z_{n} \delta-Q_{n}$.

This is proven for irreducible varieties in [11, Lemma 4, p. 37 or Theorem 5, p. 77]. The same proof works for varieties of pure dim:

Recall from $\S 1$ that ${ }_{n} \mathcal{O}_{p} / I(V, p)$ is finitely generated over ${ }_{r} \mathcal{O}_{p}$, i.e. for every $f \in \underset{n}{ } \mathcal{O}$,

$$
f=\sum_{a_{j}<k} f_{a}\left(z_{1}, \cdots, z_{r}\right) z_{r+1}^{a} \cdots z_{n}^{a}{ }^{n} \bmod \left(P_{r+1}, \ldots, P_{n}\right) .
$$

Then

$$
\delta^{N} f=\widetilde{R}\left(z^{\prime}, z_{r+1}\right) \bmod \left(P_{r+1}, \cdots, P_{n}, z_{r+2} \delta-Q_{r+2}, \cdots, z_{n} \delta-Q_{n}\right)
$$

where $\widetilde{R} \in{ }_{r} \Theta\left[z_{r+1}\right]$ is a pseudopolynomial, by replacing $z_{i} \delta$ by $\left(z_{i} \delta-\underline{\sim}_{j}\right)+Q_{j}$ for $j=r+2, \cdots, n$. Now applying the algebraic division algorithm to $\widetilde{R}$ and $P_{r+1}$, we have

$$
\delta^{N} f=R\left(z^{\prime}, z_{p+1}\right) \bmod \left(P_{r+1}, \cdots, P_{n}, z_{r+2} \delta-Q_{r+2}, \cdots, z_{n} \delta-Q_{n}\right)
$$

where $R \in{ }_{r} \mathcal{O}\left[z_{r+1}\right]$ is of degree $<\operatorname{deg} P_{r+1}$. Now if $f \in I(V, p)$, then $R \in I(V, p)$ so $R \equiv 0$ because it has lower degree than the minimal polynomial which is unique by Proposition 4.4.

Conversely if $\delta^{N} f$ is generated by these functions, then $\delta^{N} f \equiv 0$ on $V$; but $\left\{z \in V \mid \delta\left(z^{\prime}\right) \neq 0\right\}$ is dense in $V$ so $f \equiv 0$ on $V$.

I now sketch the proof of Oka's lemma as in [11, Theorem 4, p. 77] as it will be necessary to make some careful observations about the proof. I ignore the question of whether all the steps can be carried out in some fixed open set, which was the original motivation for the lemma.

Oka's lemma. Let $f_{1}, \cdots, f_{q} \in{ }_{n} \mathcal{O}$ and $R\left(f_{1}, \cdots, f_{q}\right)$ denote the sheaf of relations, i.e., $R$ is the submodule of ${ }_{n} \mathcal{O}^{q}$ consisting of $\left(\alpha_{1}, \cdots, a_{q}\right) \in{ }_{n} \Theta^{q}$ with $\sum_{i=1}^{q} a_{i} f_{i}=0$. Then $R$ is finitely generated over ${ }_{n} \Theta$.

The proof is by induction on $n$. The relations of $f_{1}, \cdots, f_{q}$ will be reduced to several relations of the type $R\left(g_{1}, \cdots, g_{q}\right)$ in ${ }_{n-1} \Theta^{q}$.

We may clearly suppose that at least one $f_{i} \neq 0$. Since the result is local and permits multiplication by units, we can assume, after a linear change of coordinates if necessary, that each $f_{i}$ is a pseudopolynomial, 


$$
f_{i}=\sum_{\nu=0}^{k} a_{\nu}^{i}\left(z_{1}, \ldots, z_{n-1}\right) z_{n}^{\nu} \text { where } a_{\nu}^{i} \epsilon_{n-1} \Theta
$$

and at least one $f_{i}$, say $f_{q}$, is a Weierstrass polynomial of degree $k$. A relation $\left(a_{1}, \cdots, a_{q}\right) \in \mathcal{R}$ is said to be a polynomial relation if each $\alpha_{i} \epsilon_{n-1} \mathcal{O}\left[z_{n}\right]$; then $R$ is generated over ${ }_{n} \mathcal{O}$ by the polynomial relation.

Let $\left(\alpha_{1}, \cdots, \alpha_{q}\right) \in R$ and for each $i=1, \cdots, q-1$, write $\alpha_{i}=\mu_{i} f_{q}+r_{i}$ by the division theorem, where $\mu_{i} \in{ }_{n} \mathcal{O}$ and $r_{i} \in{ }_{n-1} \mathcal{O}\left[z_{n}\right]$ has degree $<\operatorname{deg} f_{q} \cdot$ Let $r_{q}$ be defined by the equations:

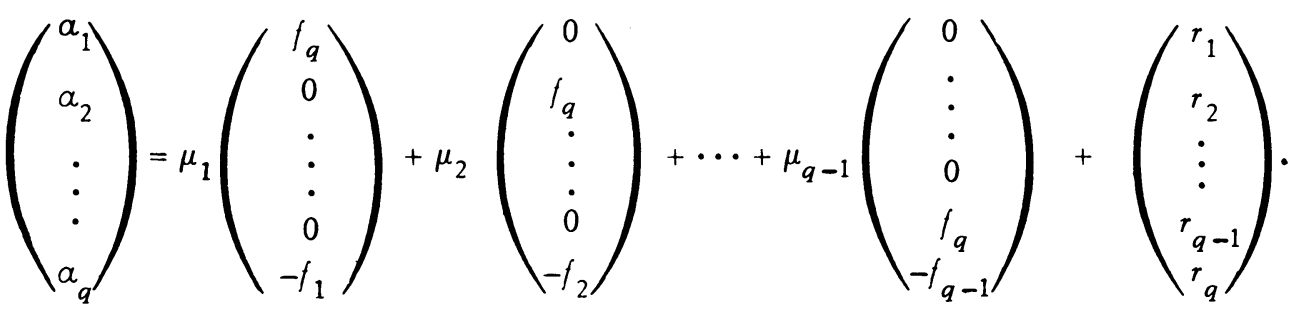

It remains only to show that $r_{q}$ is a pseudopolynomial. Clearly $\left(r_{1}, \cdots, r_{q}\right) \in R$ so $r_{q} f_{q}=-\Sigma_{i<q}{ }_{i} f_{i} \epsilon_{n-1} \Theta\left[z_{n}^{q}\right]$. By the algebraic division algorithm $r_{q} f_{q}^{q}=$ $Q f_{q}+R$ where $Q, R \epsilon_{n-1} O\left[z_{n}\right]$ and $\operatorname{deg} R<\operatorname{deg} f_{q}$. But then $R / f_{q}$ is holomorphic, $f_{q}$ vanishes to order $\operatorname{deg} f_{q}$ in the $z_{n}$ direction because it is Weierstrass, and $R$ vanishes to lower order. Thus $R \equiv 0$ and $r_{q}=Q$.

Remark 1. The first $q-1$ entries of the above relations are all of degree $\leq \operatorname{deg} f_{q}$. However, little can be said about the degree of the $q$ th entries of these relations other than they have degree $\leq \max _{1 \leq i \leq q} \operatorname{deg} f_{i}=K$. In the application to the structure sheaf of a variety, $f_{q}$ will always be picked to have degree $\leq \mu(V, p)$.

Next we show that there exist finitely many polynomial relations $\pi=\left(\pi_{1}, \cdots\right.$,

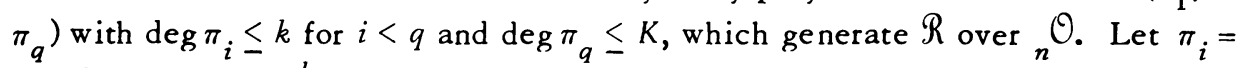
$\Sigma_{\nu} c_{\nu}^{i} z_{n}^{\nu}$ and $f_{i}=\Sigma_{\nu}^{k_{i}} a_{\nu}^{i} z_{n}^{\nu}$; then $\pi$ is a relation if and only if

$$
\sum_{i=1}^{q} \sum_{j=0}^{\nu} a_{\nu-j}^{i} c_{j}^{i}=0 \text { in }{ }_{n-1} \mathcal{O} \text { for } \nu=0, \ldots, K+k
$$

This means the element

$$
\left[c_{\nu}^{i}\right]=\left(c_{0}^{1}, \cdots, c_{k}^{1}, c_{0}^{2}, \cdots, c_{k}^{2}, \cdots, c_{0}^{q-1}, \ldots, c_{k}^{q-1}, c_{0}^{q}, \ldots, c_{K}^{q}\right) \epsilon_{n-1} \Theta^{m},
$$

$m=(k+1)(q-1)+K+1$, is a relation between the finitely many sections

$$
s_{\nu}=\left(a_{\nu}^{1}, \ldots, a_{\nu-k}^{1}, a_{\nu}^{2}, \ldots, a_{\nu-k}^{2}, \ldots, a_{\nu}^{q-1}, \ldots, a_{\nu-k}^{q-1}, a_{\nu}^{q}, \cdots, a_{\nu-K}^{q}\right),
$$


where $a_{\nu}^{i}=0$ if $\nu<0$. Thus by the induction hypothesis, there exist finitely many sections $\left[b_{\nu}^{i}\right]_{\mu} \epsilon_{n-1} \mathcal{O}^{m}$ generating these relations. That is for any $\left[c_{\nu}^{i}\right]$ as above, there exist $\psi_{\mu} \epsilon{ }_{n} \mathcal{O}$ such that $c_{\nu}^{i}=\Sigma_{\mu} \psi_{\mu} b_{\nu \mu}^{i}$ for all $i$ and $\nu$. Thus

$$
\pi=\left[\pi_{i}\right]=\left[\sum_{\nu} c_{\nu}^{i} z_{n}^{\nu}\right]=\sum_{\mu} \psi_{\mu}\left[\sum b_{\nu \mu}^{i} z_{n}^{\nu}\right]
$$

Remark 2. Each $s_{\nu}$ has as entries either all the tail coefficients $a_{0}^{i}$ or $a_{k}^{q}=1$. (For $\nu \leq k$, it contains $a_{0}^{i}$ and for $\nu \geq k$, it contains $a_{k}^{q}$.)

Remark 3. There is no mixing of terms with respect to $i$ in equations (*), e.g., only $a^{i}$ terms are multiplied times $c^{i}$ terms.

Now we apply this method to the relations in ${ }_{n} \mathcal{O}^{2(n-r)}$ between $\delta^{N}$, $z_{r+2} \delta-Q_{r+2}, \cdots, z_{n} \delta-Q_{n}, P_{r+1}, \cdots, P_{n}$ to reduce it to some other relations of functions of less variables and repeat the process on each of these relations until we come down to $(2 k)^{n-r}$ relations of $2(n-r)(k+1)^{n-r}$ functions of $r$ variables. Indeed, during the step from a relation in ${ }_{n} \mathcal{O}^{2(n-r)}$ to $2 k$ relations in ${ }_{n-1} \mathcal{O}^{2(n-r)(k+1)}$, use $P_{n}$ as the Weierstrass element $f_{q}$ to divide by in the reduction to polynomial relations; $P_{n+1}, \cdots, P_{n-1}, z_{r+2} \delta-Q_{r+2}, \cdots, z_{n-1} \delta-$ $Q_{n-1}$ are all of degree zero in $z_{n}$ and $z_{n} \delta-Q_{n}$ is of degree one in $z_{n}$ so $k=K$. By Remark 2, each resulting section so contains either $P_{r+1}, \cdots$, and $P_{n}$ or $1-$ in any event, some entry whose degree in $z_{n-1}$ is $\leq \mu(V, p)$. In the next stage the choice of special element to reduce the section depends upon $\nu$ : for $\nu \leq k$, use $P_{n-1}$; for $\nu \geq k+1$, use 1 . Again each resulting section will contain some entry whose degree in $z_{n-2}$ is $\leq \mu(V, p)$, etc.

The special results for $r=1$ and 2 arise because relation ideals in ${ }_{1} \mathcal{O}$ and ${ }_{2} \mathcal{O}$ are essentially trivial.

Proposition 4.5. Let $\lambda:{ }_{2} \Theta^{l} \rightarrow{ }_{2} \mathcal{O}^{\prime}$ be an ${ }_{2} \mathcal{O}^{\prime}$-bomomorphism, then \# ker $\lambda \leq l$.

Proof (as in [9, Proposition 8]). Consider the exact sequence

$$
\operatorname{Ker} \lambda \rightarrow{ }_{2} \Theta^{l} \stackrel{\lambda}{\longrightarrow}{ }_{2} \Theta^{i} \rightarrow{ }_{2} \Theta^{l^{\prime}} / \mathrm{im} \lambda \rightarrow 0 .
$$

According to the Hilbert-Syzygy theorem [7, p. 74], $\operatorname{Ker} \lambda$ is a free ${ }_{2}{ }$ module. Since $\operatorname{Ker} \lambda$ is a free submodule of ${ }_{2} \mathcal{O}^{l}, \# \operatorname{Ker} \lambda \leq l$.

Proposition 4.6. Let $\lambda:{ }_{1} \mathcal{O}^{l} \rightarrow{ }_{1} \mathcal{O}^{\prime}$ be a ${ }_{1} \mathcal{E}$ bomomorphism, then \#Ker $\lambda \leq l$.

Proof. First suppose $l^{\prime}=1$ and $\lambda=\left(\lambda_{1}(z), \cdots, \lambda_{l}(z)\right)$. Each $\lambda_{j}(z)$ can be written as $z^{d} j_{j}(z)$ where $u_{j}(0) \neq 0$ and by relabeling, assume that $d_{1}=$ $\min \left(d_{1}, \cdots, d_{l}\right)$. Then $\left(f_{1}, \cdots, f_{l}\right) \in \operatorname{Ker} \lambda$ if and only if 


$$
z^{d}\left(u_{1} f_{1}+\sum_{j=2}^{l} z^{d_{j}-d_{1}} u_{j} f_{j}\right)=0
$$

Consequently the mapping ${ }_{1} \Theta^{l-1} \rightarrow{ }_{1} \Theta^{l}$ defined by

$$
\left(g_{2}, \ldots, g_{l}\right) \rightarrow\left(-\frac{1}{u_{1}} \sum_{j=2}^{l} z^{d_{j}-d_{1}} u_{j} g_{j}, g_{2}, \ldots, g_{l}\right)
$$

is onto $\operatorname{Ker} \lambda$.

Now induct on $l^{\prime}$ and let $\lambda$ be the $l^{\prime} \times l$ matrix $\left[\lambda_{i j}\right]$. By the case $l^{\prime}=1$, the solutions to $\Sigma_{j=1}^{l} \lambda_{1 j} f_{j}=0$ have a basis $\left(\Phi_{j}^{\mu}\right), \mu=1, \cdots, l$. Now the general solution $\left(f_{j}\right) \in \operatorname{Ker} \lambda$ has the form $f_{j}=\Sigma_{\mu} \psi_{\mu} \Phi_{j}^{\mu}$ and satisfies the 2 nd through $l$ th equations, so

$$
0=\sum_{j} \lambda_{i j} \sum_{\mu} \psi_{\mu} \Phi_{j}^{u}=\sum_{\mu} \psi_{\mu}\left(\sum_{j} \lambda_{i j} \Phi_{j}^{\mu}\right) \text { for } i=2, \ldots, l^{\prime}
$$

Let $\gamma_{i \mu}=\Sigma_{j} \lambda_{i j} \Phi_{j}^{\mu}$ for $i=2, \cdots, l^{\prime}$. Then by the induction hypothesis, the solutions to $0=\Sigma_{\mu} \gamma_{i \mu} \psi_{\mu}$ have a basis $\left(X_{\mu}^{\rho}\right), \rho=1, \cdots, l$, so $\psi_{\mu}=\Sigma_{\rho} w_{\rho} X_{\mu}^{\rho}$ and $\Phi_{j}=\Sigma_{\rho} w_{\rho} \Sigma_{\mu} X_{\mu}^{\rho} \Phi_{j}^{\mu}$ so $\left(\Sigma_{\mu} X_{\mu}^{\rho_{\mu}} \Phi_{j}^{\mu}\right)$ form a basis of $\operatorname{Ker} \lambda$.

Now returning to the more general case of $r \geq 3$, the initial relation in ${ }_{n} \mathcal{O}^{2(n-r)}$ was reduced to $(2, k)^{n-r}$ relations in ${ }_{r} \mathcal{O}^{2(n-r)(k+1)^{n-r}}$, whose entries are just the coefficients of $\delta^{N}, z_{r+2} \delta-Q_{r+2}, \cdots, z_{n} \delta-Q_{n}, P_{r+1}, \cdots, P_{n}$. These coefficients have been rigged to be either identically zero or regular in each of the directions $z_{1}, \cdots, z_{r}$, so we can continue the process of reducing to relations of less variables without making any change of coordinates. There is a new difficulty: whereas previously we had a bound on the length of the resulting sections because $k=K$, we now have no such bound. However we are not really interested in the length of the entire section, but only in the number of terms $c_{\nu}^{1}$ that will eventually be used to generate $\pi_{1}$ 's such that $\delta^{N} \pi_{1}+\left(z_{r+2} \delta-Q_{r+2}\right) \pi_{2}+$ $\cdots+\left(z_{n} \delta-Q_{n}\right) \pi_{n-r}+P_{r+1} \pi_{n-r+1}+\cdots+P_{n} \pi_{2(n-r)}=0$, and it is poss ible to put a bound on these. Each resulting relation contains either some tail coefficient or 1-hence some entry arising from the decomposition of $P^{\prime} s$, whose degree in the next variable $\leq \mu(V, p)$. These special entries can be used as the Weierstrass element in the next stage, so by Remark 1, the number of terms used to generate $\pi_{1}$ grows only by a multiplicative factor of $k+1$ each time. We continue until reaching ${ }_{0} O=\mathrm{C}$ and read off the dimension of the appropriate vector space.

Remark. If $V$ is an algebraic variety, the generators resulting from this proof are polynomials. 
The following example of one-dimensional analytic variety in $\mathrm{C}^{3}$ whose global ideal is not finitely generated was communicated to me by Bernard Kripke. There is, of course, no such example in $\mathrm{C}^{1}$ or $\mathrm{C}^{2}$ since any subvariety of $\mathrm{C}^{1}$ or $\mathrm{C}^{2}$ is generated locally by one or two elements.

In $\mathrm{C}^{3}$, there is, for every $n$, a variety $V$ such that $I(V)$ tequires at least $n$ generators. To construct such a variety, note that there are $(n+1)(n+2) / 2$ monomials in the three variables $x, y, z$ of degree $n$, e.g. for $n=2$, they are $x^{2}$, $x y, x z, \mathrm{y}^{2}, y z, z^{2}$. Thus the vector space $H_{n}$ of homogeneous polynomials of degree $n$ on $\mathrm{C}^{3}$ has dimension $k=(n+1)(n+2) / 2$ and is spanned by homogeneous polynomials $p_{1}, \cdots, p_{k}$. There is no dependence relation of the form $c_{1} p_{1}+\cdots$ $+c_{k} p_{k} \equiv 0$, or in other words, the map: $\mathbf{C}^{3} \rightarrow \mathbf{C}^{k}$ defined by $(x, y, z) \rightarrow$ $\left(p_{1}(x, y, z), \cdots, p_{k}(x, y, z)\right)$ maps $\mathbf{C}^{3}$ into no complex hyperplane in $\mathbf{C}^{k}$. Let $u=(x, y, z)$; we can find $u_{1}, \cdots, u_{k}$ such that the vectors $\left(p_{1}\left(u_{1}\right), \cdots, p_{k}\left(u_{1}\right)\right)$, $\cdots,\left(p_{1}\left(u_{k}\right), \cdots, p_{k}\left(u_{k}\right)\right)$ are linearly independent in $C^{k}$. The rows and columns of the $k \times k$ matrix $\left[p_{i}\left(u_{j}\right)\right]$ are independent so if $f=c_{1} p_{1}+\cdots+c_{k} p_{k}$, then $\left(f\left(u_{1}\right), \cdots, f\left(u_{k}\right)\right)=0 \Leftrightarrow\left(c_{1}, \cdots, c_{k}\right)=0$.

In other words, every homogeneous polynomial of degree $n$ which vanishes at $u_{1}, \cdots, u_{k}$ vanishes identically. A fortiori, every homogeneous polynomial of degree $<n$ which vanishes at $u_{1}, \cdots, u_{k}$ must vanish identically. Now let $V$ be the variety consisting of the $k$ complex lines joining the origin to the points $u_{1}, \cdots, u_{k}$ in $\mathbf{C}^{3}$. If $f$ is any holomorphic function in $I(V)$, then $f$ can be expanded in homogeneous polynomials $f(x, y, z)=\Sigma_{j=0}^{\infty} b_{j}(x, y, z)$, where $b_{j}$ is a homogeneous polynomial of degree $j$. Since $f(t x, t y, t z)=\sum_{j=0}^{\infty} t^{j} b_{j}(x, y, z)$, it follows that each of the $b_{j}$ vanish on $V$ as well. Therefore $f=\Sigma_{j=n+1}^{\infty} b_{j}$. Indeed, every holomorphic function which vanishes on the intersection of $V$ with a neighborhood of the origin is a sum of homogeneous polynomials of degree $>n$.

Now there are $k+n+2$ homogeneous monomials of degree $n+1$ on $\mathbf{C}^{3}$, say $q_{1}, \cdots, q_{k+n+2}$. If $b \in H_{n+1}$, then $b \in I(V) \Leftrightarrow b\left(u_{1}\right)=\cdots=b\left(u_{k}\right)=0$. Consider the system of linear equations:

$$
\begin{aligned}
& c_{1} q_{1}\left(u_{1}\right)+\cdots+c_{k+n+2} q_{k+n+2}\left(u_{1}\right)=0, \\
& c_{1} q_{1}\left(u_{k}\right)+\cdots+c_{k+n+2} q_{k+n+2}\left(u_{k}\right)=0 .
\end{aligned}
$$

It defines a subspace of $\mathrm{C}^{k+n+2}$ of dimension $\geq k+n+2-k=n+2$ corresponding to a subspace $S$ of $H_{n+1}$ consisting of homogeneous polynomials $b=c_{1} q_{1}+\cdots$ $+c_{k+n+2} q_{k+n+2}$ which vanish on $V$. There are at least $n+2$ linearly independent homogeneous polynomials of degree $n+1$ which vanish on $V$; since every element of $I(V)$ is a sum of homogeneous polynomials of degree $\geq n+1$, it follows 
that any set of generators for $I(V)$ must contain at least $n+2$ elements. Suppose $g_{1}, \cdots, g_{m}$ generate $I(V)$ and write $g_{i}=b_{i}+r_{i}$, where $b_{i} \in H_{n+1}$ and $r_{i}$ is of order $>n+1$. If $b \in H_{n+1}$ and $b=f_{1} g_{1}+\cdots+f_{m} g_{m}$ where $f_{i} \in \underset{n}{\mathcal{O}_{0}}$, then $b=$ $f_{1}(0) b_{1}+\cdots+f_{m}(0) b_{m}$. That is, the vector space $S=H_{n+1} \cap I(V)$ is spanned by $b_{1}, \cdots, b_{m}$ so $m \geq n+2$.

Now this proves that there is no bound on the number of generators that may be required for the ideal of a variety in $\mathrm{C}^{3}$. Let $V_{1}, V_{2}, V_{3}, \cdots$ be a sequence of varieties in $\mathrm{C}^{3}$ constructed in this way so that $I\left(V_{j}\right)$ requires at least $j+2$ generators and each $V_{j}$ consists of $(j+2)(j+1) / 2$ lines through the origin. Now choose inductively a sequence of vectors $c_{1}, c_{2}, c_{3}, \cdots$ in $\mathrm{C}^{3}$ as follows: let $c_{1}=0$ and suppose $c_{1}, \cdots, c_{n}$ have already been chosen. Each $V_{j}$ for $j=1, \cdots$, $n+1$ consists of a union of complex lines through the origin spanned by a finite set $S_{j}$ of vectors; let $A_{n}=S_{1} \cup \cdots \cup S_{n+1}$. Each pair of vectors in $A_{n}$ span a complex 2-dim subspace of $\mathrm{C}^{3}$; let $B_{n}$ be the union of all such subspaces and let $E_{n}=\left(B_{n}+c_{1}\right) \cup\left(B_{n}+c_{2}\right) \cup \cdots \cup\left(B_{n}+c_{n}\right)$ be the union of the translates of $B_{n}$ through the vectors $c_{1}, \cdots, c_{n}$. Now choose $c_{n+1}$ so that $\operatorname{dist}\left(c_{n+1}, E_{n}\right) \geq 1$.

Let $W_{j}=V_{j}+c_{j}$ for $j=1, \cdots, n+1$ and it follows that $\operatorname{dist}\left(W_{n+1}, W_{1} \cup \cdots \cup W_{n}\right) \geq 1$. Indeed, there are $u_{n+1} \in W_{n+1}$ and $u_{j} \in W_{j}$ for some $1 \leq j \leq n$ such that $\operatorname{dist}\left(W_{n+1}, W_{1} \cup \cdots \cup W_{n}\right)=\left\|u_{n+1}-u_{j}\right\|$. Say $u_{n+1}=$ $c_{n+1}+t a$ for some $a \in S_{n+1}$ and $u_{j}=c_{j}+s b$ for some $b \in S_{j}$. Then $\left\|u_{n+1}-u_{j}\right\|=$ $\left\|c_{n+1}-\left(c_{j}-t a+s b\right)\right\|$, but $c_{j}-t a+s b \epsilon c_{j}+B_{n} \subset E_{n}$. Thus $\left\|u_{n+1}-u_{j}\right\| \geq$ $\operatorname{dist}\left(c_{n+1}, E_{n}\right) \geq 1$.

Finally let $W=\bigcup_{j=1}^{\infty} W_{j}$; by construction this union is locally finite so $W$ is a variety in $\mathrm{C}^{3}$. Clearly no finite subset of $\Theta\left(\mathrm{C}^{3}\right)$ can generate $I(W)$ since at least $n$ functions are required just to generate the germ of $I(W)$ at $c_{n}$.

Since $W$ can be embedded in $\mathbf{C}^{n}$ for every $n>3$, it is also true that for every $n \geq 3$, there is a variety $W \subset \mathrm{C}^{n}$ such that $I(W)$ is not finitely generated.

Note that $\mu\left(W, c_{n}\right)=m\left(W, c_{n}\right)=(n+1)(n+2) / 2$ and that the minimal and maximal multiplicity are one at all other points of $W$.

The following construction of an irreducible one-dimensional variety in $\mathrm{C}^{3}$ whose global ideal is not finitely generated was suggested by James King:

For any integer $n$, there exists an irreducible space curve $X_{n}$ [17] whose ideal cannot be generated by fewer than $n$ elements. Then these $X_{n}$ can be patched together away from the singular points to form a noncompact, irreducible, one-dimensional complex space $X$. By [7, Theorem IX, B.10], $X$ is a Stein space so [7, Theorem VII, C.10] there is a holomorphic homeomorphism of $X$ into $\mathrm{C}^{3}$. The obstruction to the existence of an imbedding of $X$ in $\mathrm{C}^{3}$ is that the local holomorphic imbedding dimension of $X$ be bounded [18]; but this is at most 3, so an embedding exists. 


\section{BIBLIOGRAPHY}

1. J. Becker, Continuing analytic sets across $\mathbf{R}^{n}$, Math. Ann. 195 (1972), 103-106.

2. E. Bishop, Mappings of partially analytic spaces, Amer. J. Math. 83 (1961), 209242. MR 23 \#A1054.

3. - Some global problems in the theory of functions of several complex variables, Amer. J. Math. 83 (1961), 479-498. MR 25 \#4131. \#5191.

4. - Partially analytic spaces, Amer. J. Math. 83 (1961), 669-692. MR 25

5. O. Forster and K. J. Ramspott, Über die Darstellung analytischer Mengen, Bayer. Akad. Wissenschaften Math.-Natur. K1. S.-B. 1963, 89-99 (1964). MR $29 \# 4912$.

6. R. C. Gunning, Lectures on complex analytic varieties: The local parametrization theorem, Princeton Univ. Press, Princeton, N. J.; Univ. of Tokyo Press, Tokyo, 1970. MR $42 \# 7941$.

7. R. C. Gunning and H. Rossi, Analytic functions of several complex variables, Prentice-Hall Series in Modern Analysis, Prentice-Hall, Englewood Cliffs, N. J., 1965. MR 31 \#4927.

8. H. Grauert, Charakterisierung der holomorphvollständiger komplexen Räume, Math. Ann. 129 (1955), 233-259. MR 17, 80.

9. B. Kripke, Finitely generated coherent analytic sheaves, Proc. Amer. Math. Soc. 21 (1969), 530-534. MR $39 \# 1681$.

10. D. Mumford, Introduction to algebraic geometry, Harvard Univ. Press, Cambridge, Mass.

11. R. Narasimhan, Introduction to the theory of analytic spaces, Lecture Notes in Math., no. 25, Springer-Verlag, Berlin and New York, 1966. MR 36 \#428.

12. R. Remmert, Holomorphe and meromorphe Abbildungen komplexer Räume, Math. Ann. 133 (1957), 328-370. MR 19, 1193.

13. W. Rudin, A geometric criterion for algebraic varieties, J. Math. Mech. 17(1967/68), 671-683. MR 36 \#2829.

14. H. Whitney, Local properties of analytic varieties, Differential and Combinatorial Topology, Princeton Univ. Press, Princeton, N. J., 1965. MR 32 \#5924.

15. - Tangents to an analytic variety, Ann. of Math. (2) 81 (1965), 496-549. MR 33 \#745.

16. - Complex analytic varieties, Addison-Wesley, Reading, Mass., 1972.

17. F. S. Maculy, Algebraic theory of modular systems, Cambridge, Mass., 1916.

18. R. Narasimhan, Imbeddings of holomorphically complete complex spaces, Amer. J. Math. 82 (1960), 917-934. MR $26 \# 6438$.

DEPARTMENT OF MATHEMATICS, STATE UNIVERSITY OF NEW YORK AT ALBANY, ALBANY, NEW YORK 12222

Current address: Department of Mathematical Sciences, Purdue University, West Lafayette, Indiana 47907 